Research Article

\title{
Optimal Time-Consistent Investment and Reinsurance Strategies with Default Risk and Delay under Heston's SV Model
}

\author{
Sheng Li $(\mathbb{D})$ and Zhijian Qiu \\ School of Economic Mathematics, Southwestern University of Finance and Economics, Chengdu 611130, China \\ Correspondence should be addressed to Sheng Li; 1180202z1002@smail.swufe.edu.cn
}

Received 13 September 2020; Revised 17 February 2021; Accepted 24 February 2021; Published 13 March 2021

Academic Editor: Bekir Sahin

Copyright ( 92021 Sheng Li and Zhijian Qiu. This is an open access article distributed under the Creative Commons Attribution License, which permits unrestricted use, distribution, and reproduction in any medium, provided the original work is properly cited.

\begin{abstract}
Considering the influence of past information on the decision-making of insurers, the correlation between the insurance businesses owned by insurers, and the possible default faced by insurers, we investigate the mean-variance investment and reinsurance problem with the default risk, delay, and common shock dependence. We characterize the insurance market by twodimensional dependent claims, the financial market by the Heston SV model, and default risk by reduced-form approach and then obtain the evolution equation of the insurer's wealth. Based on the introduction of time delay, the insurer's wealth dynamics characterized by a stochastic delay differential equation are obtained. Furthermore, applying stochastic control theory within the game-theoretic framework and stochastic control theory with delay, we derive optimal time-consistent investment and reinsurance strategies, as well as equilibrium value function and equilibrium efficient frontier. Finally, we use a numerical example to analyze the influence of parameters on the time-consistent equilibrium strategies and give an economic explanation.
\end{abstract}

\section{Introduction}

The core problem of the insurance company is risk management, which is to manage and control the surplus process of the insurance company. Combining actuarial problems with portfolio problems, we should pay attention to not only the risks and benefits of investment but also the risks that the company underwrites to ensure that it can pay the claims of the insured on time. Therefore, in recent years, the investment and reinsurance of insurance companies have attracted a lot of attention. Browne [1] first studies the optimal investment strategy under the goal of maximizing the expected utility of an exponential utility function and minimizing ruin probability by simulating insurers' surplus process with geometric Brownian motion. Yang and Zhang [2] also consider the optimal investment problem under the jump-diffusion model for the expected utility maximization. Bai and Guo [3] study the optimal investment strategy for insurers to invest in multiple risky assets under the diffusion risk model. Hipp and Plum [4] investigate the investment strategy to minimize the ruin probability of insurance companies in the classical Cramer-Lundberg (CL) model. Many pieces of literature have also studied the reinsurance problem from different perspectives. Kaluszka [5] considers the optimal reinsurance strategy under the mean-variance criterion. Højgaard and Taksar [6] study the proportional reinsurance problem of the maximum return function under the diffusion risk model, which approximates expressions of the optimal strategy and the value functions are obtained. Cao and Wan [7] derive the investment and reinsurance strategies that maximize the exponential utility of terminal wealth under the diffusion approximation (DA) model by combining investment and reinsurance. Moreover, Zhang et al. [8] consider investment and reinsurance problems with transaction costs. Gu et al. [9] study the optimal proportional reinsurance and investment of insurance companies under the CEV model. Zhao et al. [10] study the optimal excess-of-loss reinsurance and investment problem under the Heston stochastic volatility model.

It is well known that the mean-variance optimization problem is time-inconsistent in the sense that it does not follow the Bellman optimality principle. In the framework of 
game theory, Bjork and Murgoci [11] and Björk et al. [12] establish a stochastic control theory for solving time-inconsistent optimization problems. Under this theory, the obtained control strategy is time-consistent, which is not only optimal at present but also optimal in the future with the passage of time. Therefore, there has been an increased interest in finding a time-consistent equilibrium strategy for the mean-variance investment and reinsurance problem in recent years. Zeng and Li [13] consider an optimal investment-reinsurance problem and obtain a closed-form expression for time-consistent investment and reinsurance strategy. Li et al. [14] derive a time-consistent investmentreinsurance strategy under Heston's SV model. Liang and Song [15] arrive at an explicit time-consistent investmentreinsurance strategy under partial information.

There are two deficiencies in the abovementioned literature, which are worthy of further discussion. On the one hand, they are based on the assumption that different insurance businesses are independent of each other. However, in practice, different insurance businesses of insurers often interact with each other in a certain way. For example, the outbreak of the 2019-nCoV may lead to the simultaneous occurrence of medical claims and death claims. In order to describe the interdependence between different insurance businesses, a risk dependence model is proposed. In this respect, Bai et al. [16] obtain the optimal excess-of-loss reinsurance strategy by minimizing the ruin probability in the corresponding DA model. Liang and Yuen [17] adopt the variance premium principle and consider the optimal proportional reinsurance problem under the CL model and DA model. Yuen et al. [18] investigate the optimal reinsurance under the expected premium principle, and the insurer's surplus process was characterized by the DA model with multiple dependent risks. Ming et al. [19] also studied the mean-variance reinsurance problem under the DA model and obtained the corresponding optimal results. $\mathrm{Bi}$ et al. [20] and Bi and Chen [21] combine investment with reinsurance and consider the investment-reinsurance problem with common shocks. Another important aspect is that the control problems considered in the above literature are based on current information to determine the optimal strategy, without considering past information. In fact, the information in the past always affects the decision-making of the insurer or investor more or less. For example, when investing in stocks, investors pay attention not only to the current stock price but also to the stock price trend in the past period. Taking past information into account in the model helps us make more reasonable decisions. Elsanousi et al. [22], Økendal and Sulem [23], David [24], and Federico et al. [25] have provided a relevant theoretical basis for solving stochastic control problems with delay by applying dynamic programming principle or stochastic maximum principle. Based on these theories, Chang et al. [26] consider the investment and consumption problem with delay on the assumption that the financial market has bounded memory. Federico [27] studies pension management by assuming that there is performance-related capital inflow/outflow in the wealth process. Shen and Zeng [28] study the optimal investment-reinsurance problem with time delay by using the maximum principle under the mean-variance criterion.

Inspired by the above works, considering the influence of past information on the decision-making of insurers, the correlation between the insurance businesses owned by insurers, and the possible default faced by insurers, we investigate mean-variance investment and reinsurance problem with the default risk, delay, and common shock dependence. By applying stochastic control theory with delay and stochastic control theory within the game-theoretic framework, we derive extended Hamilton-Jacobi-Bellman (HJB) equations with delay. Further, we obtain equilibrium strategies and the corresponding equilibrium value function under the postdefault and the predefault cases.

Compared with the existing literature, we think that the main contribution of this paper is the consideration of default risk, delay, and insurance risk common shock dependence under Heston's SV model. We also aim to seek time-consistent equilibrium strategies. We are trying to get close to the real economic environment that insurers are facing. When our model degenerates into a model without considering the default risk, delay, and insurance risk common shock dependence, we can obtain the result of $\mathrm{Li}$ et al. [14]. The remainder of this paper is organized as follows. Section 2 formulates the mean-variance investment and reinsurance problem with delay and common shock dependence under Heston's SV model. In Section 3, by solving the extended $\mathrm{HJB}$ equations with delay, we derive the time-consistent equilibrium investment and reinsurance strategies and the corresponding equilibrium value functions. Then, we obtain some results in special cases, such as without defaultable bond or without delay. In Section 4, we provide a numerical example to simulate the influence of relevant parameters on the equilibrium strategy and give an economic explanation. Section 5 concludes this paper.

\section{General Formulation}

In this paper, we consider a filtered complete probability space $\left(\Omega, \mathscr{F}_{,},\left\{\mathscr{F}_{t}\right\}_{t \in[0, T]}, \mathbb{P}\right)$ satisfying the usual conditions; that is, $\left\{\mathscr{F}_{t}\right\}_{t \in[0, T]}$ is right continuous and $\mathbb{P}$-complete, where $\left\{\mathscr{F}_{t}\right\}_{t \in[0, T]}$ is the information of the market available up to time $t$ and $[0, T]$ is a fixed and finite time horizon. All stochastic processes introduced below are assumed to be well defined and adapted processes in this probability space, and we assume that all stochastic processes are separable. We assume that there exists a martingale probability measure $\mathbb{Q}$ equivalent to the real-world probability measure $\mathbb{P}$, where $\mathbb{Q}$ is also called a risk-neutral probability measure. In addition, we suppose that there are no transaction costs or taxes in the financial or the insurance market, and trading can be continuously done.

Suppose that an insurer has an insurance portfolio business, which is composed of two different insurance businesses, such as medical insurance and death insurance. Suppose that the random variable $\left\{Y_{1 i}, i \geq 1\right\}$ represents the claim amount of the first type of insurance business; it is independent and has the same distribution function $F_{1}\left(y_{1}\right)$. 
$\left\{Y_{2 i}, i \geq 1\right\}$ represents the claim amount of the second type of insurance business; it is independent and has the same distribution function $F_{2}\left(y_{2}\right)$. We assume that if $y_{1} \leq 0$, then $F_{1}\left(y_{1}\right)=0$; otherwise, $0<F_{1}\left(y_{1}\right) \leq 1$. We also assume that if $y_{2} \leq 0$, then $F_{2}\left(y_{2}\right)=0$; otherwise, $0<F_{2}\left(y_{2}\right) \leq 1$. In addition, their moment generating functions $M_{Y_{1}}(\iota)$ and $M_{Y_{2}}(\iota)$ exist. The cumulative claim processes of the two insurance businesses are as follows:

$$
\begin{aligned}
& C_{1}(t)=\sum_{i=1}^{\tilde{N}_{1}(t)} Y_{1 i}, \\
& C_{2}(t)=\sum_{i=1}^{\tilde{N}_{2}(t)} Y_{2 i},
\end{aligned}
$$

where $\left\{\tilde{N}_{1}(t)\right\}_{t>0}$ and $\left\{\tilde{N}_{2}(t)\right\}_{t>0}$ represent the numbers of claims for the first and second categories of insurance business up to time $t$, respectively.

For different insurance businesses, it is assumed that they are interdependent as follows:

$$
\begin{aligned}
& \tilde{N}_{1}(t)=N_{1}(t)+N(t), \\
& \tilde{N}_{2}(t)=N_{2}(t)+N(t),
\end{aligned}
$$

where $\{N(t)\}_{t>0},\left\{N_{1}(t)\right\}_{t>0}$, and $\left\{N_{2}(t)\right\}_{t>0}$ are three independent Poisson processes; the corresponding intensities are $\lambda, \lambda_{1}$, and $\lambda_{2}$. Therefore, the total claim amount of these two types of the insurance business is

$$
C(t)=C_{1}(t)+C_{2}(t)=\sum_{i=1}^{N_{1}(t)+N(t)} Y_{1 i}+\sum_{i=1}^{N_{2}(t)+N(t)} Y_{2 i}
$$

Suppose for arbitrary $\iota \in(0, \bar{l})$ that $E\left[Y_{1 i} e^{l Y_{1 i}}\right]$ and $E\left(Y_{2 i} e^{i Y_{2 i}}\right)$ exist; and, for some $\bar{\imath} \in(0, \infty]$, there are $\lim _{-} E\left[Y_{1 i} e^{l Y_{1 i}}\right] \longrightarrow \infty$ and $\lim _{-} E\left[Y_{2 i} e^{i Y_{2 i}}\right] \longrightarrow \infty$.

$\stackrel{\iota}{\imath}$ For simplicity, we defin $\overrightarrow{\mathrm{e}}^{\longrightarrow}$

$$
\begin{aligned}
& a_{1}:=E\left[C_{1}(t)\right]=\left(\lambda+\lambda_{1}\right) \mu_{11}, \\
& a_{2}:=E\left[C_{2}(t)\right]=\left(\lambda+\lambda_{2}\right) \mu_{21}, \\
& b_{1}^{2}:=\operatorname{Var}\left[C_{1}^{2}(t)\right]=\left(\lambda+\lambda_{1}\right) \mu_{12}, \\
& b_{2}^{2}:=\operatorname{Var}\left[C_{2}^{2}(t)\right]=\left(\lambda+\lambda_{2}\right) \mu_{22},
\end{aligned}
$$

where $\mu_{11}=E\left[Y_{1 i}\right], \quad \mu_{12}=E\left[Y_{1 i}^{2}\right], \quad \mu_{21}=E\left[Y_{2 i}\right], \quad$ and $\mu_{22}=E\left[Y_{2 i}^{2}\right]$. The surplus process of the insurer up to time $t$ is modeled by

$$
R(t)=R_{0}+c_{0} t-C(t),
$$

where $R_{0}$ is the initial surplus, $c_{0}$ is the premium rate, and $C(t)$ is the cumulative claim process up to time $t$. We assume that insurance premium rate at time $t$ is calculated by the expected value principle; that is, $c_{0}=\left(1+\theta_{1}\right) a_{1}+\left(1+\theta_{2}\right) a_{2}$, where $\theta_{1}$ and $\theta_{2}$ are the safety loadings of the insurer for the first class claims and second class claims, respectively.

In addition, insurer can continuously purchase proportional reinsurance. $q_{1}(t)$ and $q_{2}(t)$ represent the insurer's retention level after purchasing reinsurance, respectively.
That is to say, the insurer pays $q_{1}(t) Y_{1 i}$ (or $\left.q_{2}(t) Y_{2 i}\right)$ of a claim occurring at time $t$ and the reinsurer pays $1-q_{1}(t) Y_{1 i}$ (or $\left.1-q_{2}(t) Y_{2 i}\right)$. Let the reinsurance premium also be calculated by the expected value principle. For the new business, the premium has to be paid at rate $\left(1-q_{1}(t)\right)\left(1+\eta_{1}\right) a_{1}+\left(1-q_{2}(t)\right)\left(1+\eta_{2}\right) a_{2}$, where $\eta_{1}$ and $\eta_{2}$ are the safety loadings of the reinsurer for the first class claims and second class claims, respectively. Without loss of generality, we assume that $\eta_{1}>\theta_{1}$ and $\eta_{2}>\theta_{2}$. Note that, for the insurer, $q_{1}(t) \in[0,1]\left(q_{2}(t) \in[0,1]\right)$ corresponds to a reinsurance cover and $q_{1}(t)>1\left(q_{2}(t)>1\right)$ would mean that the company can take an extra insurance business from other companies (i.e., act as a reinsurer for other cedents). After reinsurance, the premium of the insurer is given by

$$
\begin{aligned}
c & =c_{0}-\left[\left(1-q_{1}(t)\right)\left(1+\eta_{1}\right) a_{1}+\left(1-q_{2}(t)\right)\left(1+\eta_{2}\right) a_{2}\right] \\
& =\left[\left(1+\eta_{1}\right) q_{1}(t)+\theta_{1}-\eta_{1}\right] a_{1}+\left[\left(1+\eta_{2}\right) q_{2}(t)+\theta_{2}-\eta_{2}\right] a_{2} \\
& =\left(1+\eta_{1}\right) q_{1}(t) a_{1}+\xi_{1} a_{1}+\left(1+\eta_{2}\right) q_{2}(t) a_{2}+\xi_{2} a_{2},
\end{aligned}
$$

where $\xi_{1}=\theta_{1}-\eta_{1}$ and $\xi_{2}=\theta_{2}-\eta_{2}$.

Then the reserve process $\{R(t)\}_{t \geq 0}$ of the insurer is

$$
\mathrm{d} R(t)=c \mathrm{~d} t-q_{1}(t) \mathrm{d} C_{1}(t)-q_{2}(t) \mathrm{d} C_{2}(t) .
$$

We consider a financial market consisting of a risk-free asset, a defaultable bond, and a risky asset, in which financial assets can be traded continuously. The dynamic evolution of a risk-free asset price is given by

$$
\left\{\begin{array}{l}
\mathrm{d} S_{0}(t)=r S_{0}(t) \mathrm{d} t, \quad t \in[0, T] \\
S_{0}(0)=1,
\end{array}\right.
$$

where $r(>0)$ is the interest rate of the risk-free asset. The price process of the risky asset is described by the following process:

$$
\left\{\begin{array}{l}
\mathrm{d} S(t)=S(t)\left[(r+\delta v(t)) \mathrm{d} t+\sqrt{v(t)} \mathrm{d} W_{1}(t)\right], \quad S(0)=S_{0}, \\
\mathrm{~d} v(t)=\kappa(\alpha-v(t)) \mathrm{d} t+\sigma \sqrt{v(t)} \mathrm{d} W_{2}(t), \quad v(0)=v_{0},
\end{array}\right.
$$

where $\delta, \kappa, \alpha$, and $\sigma$ are all positive constants; $W_{1}(t)$ and $W_{2}(t)$ are two standard Brownian motions with $E\left[W_{1}(t) W_{2}(t)\right]=\rho t, \rho \in[-1,1]$. In addition, we assume that $2 \kappa \alpha>\sigma^{2}$ to ensure that $v(t) \geq 0$ almost surely.

Different from the price dynamics of the risk-free asset and risky asset which are defined under the real-world probability measure $\mathbb{P}$ directly, the price process of the defaultable bond is firstly given by the martingale probability measure $\mathbb{Q}$ and then turned into the price dynamics under the probability measure $\mathbb{P}$. Following Bielecki and Jang [29], the definition of the default process is given by $Z(t)=\mathbf{1}_{\{\tau \leq t\}}$, where $\tau$ is a nonnegative random variable representing the default time of the company issuing the bond, and $\mathbf{1}$ is an indicator that takes value of one if the default occurs and zero otherwise. That is, the default process $Z(t)$ is nondecreasing right continuous and has a jump at the random time $\tau$. Furthermore, according to Driessen [30] and Duffie and Singleton [31], $Z(t)$ is supposed as the default process with a 
constant intensity $k^{\mathbb{P}}$ under the probability measure $\mathbb{P}$. Then the martingale default process $K(t)$ under probability measure $\mathbb{P}$ is given by $K(t):=Z(t)-\int_{0}^{t}(1-Z(u-)) k^{\mathbb{P}} \mathrm{d} u$, where its differential form is $\mathrm{d} K(t)=\mathrm{d} Z(t)-k^{\mathbb{P}}(1-Z(t-)) \mathrm{d} t$.

Then the price process of the defaultable bond under the martingale probability measure $\mathbb{Q}$ is considered. Similar to Bielecki and Jang [29], the defaultable bond is assumed as a zero-coupon bond with the one-unit face value and the maturity $\bar{T}$. Further, we assume that, in the event that a default occurs, the insurer recovers a fraction of the market value of the defaultable bond just prior to default and then the postdefault value of the defaultable bond is zero. If the loss rate is denoted by $\zeta$, then $1-\zeta$ is the default recovery rate. In addition, we denote by $k^{\mathbb{Q}}$ the constant intensity of the default process under martingale probability measure $\mathbb{Q}$; then $\vartheta=k^{\mathbb{Q}} \zeta$ is the risk-neutral credit spread, and then we derive the following price process of the defaultable bond under the probability measure $\mathbb{Q}$ :

$$
p(t, \bar{T})=\mathbf{1}_{\tau>t} e^{-(r+9)(\bar{T}-t)}+\mathbf{1}_{\tau \leq t}(1-\zeta) e^{-(t+9)(\bar{T}-t)} e^{r(t-\tau)},
$$

where $p(t, \bar{T})$ is a fictitious bond rather than a bond that is actually traded. That is, as Bielecki and Jang [29] and Driessen [30] mentioned, the fictitious bond allows us to take into consideration the jump risk premium in the expected return of the defaultable bond. Applying It $\widehat{o}$ formula to the above expression, we derive

$$
\mathrm{d} p(t, \bar{T})=r p(t, \bar{T}) \mathrm{d} t-\zeta e^{-(r+9)(\bar{T}-t)} \mathrm{d} K^{\mathbb{Q}}(t),
$$

where $K^{\mathbb{Q}}(t)$ is a martingale default process under the martingale probability measure $\mathbb{Q}$.

Next, we need to transform the price process of defaultable bonds under martingale probability measure $\mathbb{Q}$ into the real-world probability measure $\mathbb{P}$. Based on the above notations, by Girsanov theorem (see Kusuoka [32]), there exists a predictable process $\Delta$ such that the evolutions of the price process for the defaultable bond under realworld probability measure $\mathbb{P}$ are as follows:

$$
\mathrm{d} p(t, \bar{T})=p(t-, \bar{T})\left[r \mathrm{~d} t+(1-Z(t)) \vartheta(1-\Delta) \mathrm{d} t-(1-Z(t-)) \zeta \mathrm{d} K^{\mathbb{P}}\right],
$$

where $\Delta=\left(k^{\mathbb{P}} / k^{\mathbb{Q}}\right)$ and $K^{\mathbb{P}}=Z(t)-k^{\mathbb{Q}} \int_{0}^{t}(1-Z(u-)) \Delta \mathrm{d} u$ is an $\mathscr{F}$-martingale under probability measure $\mathbb{P}$. Similar to Duffie and Singleton [31], the probability of default under the real-world probability measure $\mathbb{P}$ is lower than that under the martingale probability measure $\mathbb{Q}$; that is, $\Delta \leq 1$. According to $\mathrm{Yu}$ [33], equation (12) consists of two components: The first component is the return of a default-free bond. The second is the difference between the risk-neutral credit spread and the real-world credit spread provided that the default has not occurred by time $t$.

Assume that $X(t)$ denotes the wealth of the insurer at time $t$, and $p_{1}(t)$ and $p_{2}(t)$ denote the amounts invested in the risky asset and defaultable bond, respectively; then $X(t)-p_{1}(t)-p_{2}(t)$ is the investment amount of a risk-free asset. Define $\pi(t)=\left(p_{1}(t), p_{2}(t), q_{1}(t), q_{2}(t)\right)$ as the investment-reinsurance strategy at time $t$; then the wealth process $\left\{X^{\pi}(t)\right\}$ of the insurer under a strategy $\pi$ satisfies the following stochastic differential equation:

$$
\begin{aligned}
\mathrm{d} X^{\pi}(t)= & \left(X^{\pi}(t)-p_{1}(t)-p_{2}(t)\right) \frac{\mathrm{d} S_{0}(t)}{S_{0}(t)}+p_{1}(t) \frac{\mathrm{d} S(t)}{S(t)}+p_{2}(t) \frac{\mathrm{d} p(t, \bar{T})}{p(t-, \bar{T})}+\mathrm{d} R(t) \\
= & {\left[r X^{\pi}(t)+\delta p_{1}(t) v(t)+p_{2}(t)(1-Z(t)) \vartheta(1-\Delta)+\left(1+\eta_{1}\right) a_{1} q_{1}(t)+\left(1+\eta_{2}\right) a_{2} q_{2}(t)\right.} \\
& \left.+\xi_{1} a_{1}+\xi_{2} a_{2}\right] \mathrm{d} t+p_{1}(t) \sqrt{v(t)} \mathrm{d} W_{1}(t)-p_{2}(t)(1-Z(t-)) \zeta \mathrm{d} M^{\mathbb{P}}(t)-q_{1}(t) d \sum_{i=1}^{N_{1}(t)+N(t)} Y_{1 i} \\
& -q_{2}(t) d \sum_{i=1}^{N_{2}(t)+N(t)} Y_{2 i} .
\end{aligned}
$$

Next, we consider the impact of historical performance on the insurer's wealth. Suppose that $f(t, X(t)-\bar{L}(t), X(t)-M(t))$ denotes the inflow/outflow of capital, then the insurer's wealth process satisfies the following stochastic delay differential equation (SDDE):

$$
\begin{aligned}
\mathrm{d} X^{\pi}(t)= & {\left[r X^{\pi}(t)+\delta p_{1}(t) v(t)+p_{2}(t)(1-Z(t)) \vartheta(1-\Delta)+\left(1+\eta_{1}\right) a_{1} q_{1}(t)+\left(1+\eta_{2}\right) a_{2} q_{2}(t)\right.} \\
& \left.+\xi_{1} a_{1}+\xi_{2} a_{2}\right] \mathrm{d} t-f(t, X(t)-\bar{L}(t), X(t)-M(t)) \mathrm{d} t+p_{1}(t) \sqrt{v(t)} \mathrm{d} W_{1}(t) \\
& -p_{2}(t)(1-Z(t-)) \zeta \mathrm{d} M^{\mathbb{P}}(t)-q_{1}(t) d \sum_{i=1}^{N_{1}(t)+N(t)} Y_{1 i}-q_{2}(t) d \sum_{i=1}^{N_{2}(t)+N(t)} Y_{2 i} .
\end{aligned}
$$


As Shen and Zeng [28] used, we describe the inflow/ outflow of capital by the following linear functions:

$$
\begin{aligned}
f(t, X(t)-\bar{L}(t), X(t)-M(t)) & =\gamma_{1}(X(t)-\bar{L}(t))+\gamma_{2}(X(t)-M(t)) \\
& =\gamma_{1}\left(X(t)-\frac{L(t)}{\int_{-h}^{0} e^{A u} \mathrm{~d} u}\right)+\gamma_{2}(X(t)-M(t)) \\
& =\left(\gamma_{1}+\gamma_{2}\right) X(t)-\bar{\gamma}_{1} L(t)-\gamma_{2} M(t),
\end{aligned}
$$

where $\gamma_{1} \geq 0$ and $\gamma_{2} \geq 0$ are constants. $\bar{\gamma}_{1}=\left(\gamma_{1} / \int_{-h}^{0} e^{A u} \mathrm{~d} u\right)$. $L(t)=\int_{-h}^{0} e^{A u} X^{\pi}(t+u) \mathrm{d} u, \quad \bar{L}(t)=\left(L(t) / \int_{-h}^{0} e^{A u}\right) \mathrm{d} u$, and $M(t)=X(t-h)$ represent the integrated, average, and point-by-point delay information during time $[t-h, t]$, respectively. $A(\geq 0)$ and $h(\geq 0)$ are given average parameter and delay parameter, respectively. Note that $\bar{L}(t)$ is defined as the weighted average value of wealth process $X(\cdot)$ in the time interval $[t-h, t]$, and the exponential decay factor $e^{A u}(u \in[-h, 0])$ denotes the weight. When $h=1, X(t)-$ $\bar{L}(t)$ and $X(t)-M(t)$ represent the average gain/loss and absolute gain/loss of the insurer in the last operation cycle, respectively. Since the inflow/outflow of capital is closely related to the past performance of the wealth process, if the past performance is good, the company will give part of the earnings to shareholders or give bonus to the management, which shows the outflow of capital; that is, $f>0$ when $X(t)>\bar{L}(t)$ and $X(t)>M(t)$. On the contrary, if the insurer's past performance is not good, the company needs additional financing to achieve the predetermined goal, which shows capital inflow; that is, $f<0$ when $X(t)<\bar{L}(t)$ and $X(t)<M(t)$. Therefore, function $f(\cdot, \cdot, \cdot)$ considers the average performance and absolute performance of insurer's wealth process in the period $[t-h, t]$.

Substituting (15) into (14), we derive

$$
\left\{\begin{array}{l}
\mathrm{d} X^{\pi}(t)=\left[\left(r-\gamma_{1}-\gamma_{2}\right) X^{\pi}(t)+\bar{\gamma}_{1} L^{\pi}(t)+\gamma_{2} M^{\pi}(t)+\delta p_{1}(t) v(t)+p_{2}(t)(1-Z(t)) \vartheta(1-\Delta)\right. \\
\left.+\left(1+\eta_{1}\right) a_{1} q_{1}(t)+\left(1+\eta_{2}\right) a_{2} q_{2}(t)+\xi_{1} a_{1}+\xi_{2} a_{2}\right] \mathrm{d} t+p_{1}(t) \sqrt{v(t)} \mathrm{d} W_{1}(t) \\
-p_{2}(t)(1-Z(t-)) \zeta \mathrm{d} M^{\mathbb{P}}(t)-q_{1}(t) d \sum_{i=1}^{N_{1}(t)+N(t)} Y_{1 i}-q_{2}(t) d \sum_{i=1}^{N_{2}(t)+N(t)} Y_{2 i}, \\
\mathrm{~d} L^{\pi}(t)=\left(X^{\pi}(t)-A L^{\pi}(t)-e^{-A h} M^{\pi}(t)\right) \mathrm{d} t .
\end{array}\right.
$$

We assume that $X(t)=X_{0}, \forall t \in[-h, 0]$, which can be interpreted as that the insurer owns wealth $X_{0}$ at time $-h$ and does not carry out any business operation within $[-h, 0]$. The integrated delay wealth initial value can be calculated by $L(0)=\left(X_{0} / A\right)\left(1-e^{-A h}\right)$.

Definition 1 (admissible strategy). For any fixed $t \in[0, T]$, an investment-reinsurance strategy $\pi=\left\{\left(p_{1}(u), p_{2}(u), q_{1}\right.\right.$ $\left.\left.(u), q_{2}(u)\right)\right\}_{u \in[t, T]}$ is said to be admissible if

(i) $\pi$ is $\left\{\mathscr{F}_{t}\right\}_{t \in[0, T]}$-progressively measurable.

(ii) $E\left[\int_{t}^{T}\left(\left|p_{1}(u)\right|^{4}+\left|p_{2} \quad(u)\right|^{4}+\left|q_{1}(u)\right|^{4}+\left|q_{2}(u)\right|^{4}\right)\right.$ $\mathrm{d} u]<+\infty$.

(iii) $\forall(x, l, v, z) \in \mathbb{R} \times \mathbb{R} \times \mathbb{R} \times\{0,1\}$, the $\operatorname{SDDE}$ (16) has a unique pathwise strong solution $\left\{X^{\pi}(u)\right\}_{u \in[t, T]}$ with $X^{\pi}(t)=x, L^{\pi}(t)=l, v(t)=v$, and $Z(t)=z$. (iv) $\forall \tau \in[1, \infty)$ and $\forall(t, x, l, v, z) \in[0, T] \times \mathbb{R} \times \mathbb{R} \times$ $\mathbb{R} \times\{0,1\}, \quad E_{t, x, l, v, z}\left[\sup _{\pi \in \Pi}\left|X^{\pi}(u)\right|^{\dagger}\right]<+\infty$, where $E_{t, x, v, z}[\cdot]$ is the conditional expectation given $X^{\pi}=x, L^{\pi}=l, v(t)=v$, and $Z(t)=z$. Let $\Pi$ be the set of all admissible investment-reinsurance strategies.

In addition, as Zhu et al. [34] discussed, we suppose throughout that $T<\bar{T}$, where $\bar{T}$ is the time of maturity of the defaultable bond. When default has occurred, that is, $\tau \leq t$, we assume that $p(t-, \bar{T})$ and fix $p_{2}(t)=0$ afterwards. We let $z$ denote initial default state, where $z=1$ and $z=0$ correspond to the postdefault cases $\tau>t$ and the predefault case $\tau \leq t$, respectively.

To take historical operating performance into account, the insurer will focus on both terminal wealth $X^{\pi}(T)$ and historical average operating performance $\bar{L}^{\pi}(T)$; hence, we 
formulate the optimal investment and reinsurance problem with delay under the mean-variance criterion as follows:

$$
J\left(t, x, l, v, z ; \pi^{*}\right)=\sup _{\pi \in \Pi}\left\{E_{t, x, l, v, z}\left[X^{\pi}(T)+\bar{\beta} \bar{L}^{\pi}(T)\right]-\frac{\omega}{2} \operatorname{Var}_{t, x, l, v, z}\left[X^{\pi}(T)+\bar{\beta} \bar{L}^{\pi}(T)\right]\right\}
$$

where risk aversion parameter $\omega$ is the risk averse coefficient. The delay parameter $\bar{\beta}(\in[0,1])$ is a constant. $E_{t, x, l, v, z}[\cdot]$ and $\operatorname{Var}_{t, x, l, v, z}[\cdot]$ represent conditional expectation and conditional variance based on $X^{\pi}(t)=x, L^{\pi}(t)=l, v(t)=v$, and $Z(t)=z$, respectively. $\bar{\beta}(\in[0,1])$ is the weight of $\bar{L}^{\pi}(t)$, indicating the degree of terminal wealth affected by historical average performance. If we write $\beta=\left(\bar{\beta} / \int_{-h}^{0} e^{A u} \mathrm{~d} u\right)$, then $X^{\pi}(t)+\bar{\beta} \bar{L}^{\pi}(t)=X^{\pi}(t)+\beta L^{\pi}(t)$, which is called the terminal wealth in the sequel. Therefore, we rewrite (17) as

$$
J\left(t, x, l, v, z ; \pi^{*}\right)=\sup _{\pi \in \Pi}\left\{E_{t, x, l, v, z}\left[X^{\pi}(T)+\bar{\beta} L^{\pi}(T)\right]-\frac{\omega}{2} \operatorname{Var}_{t, x, l, v, z}\left[X^{\pi}(T)+\bar{\beta} L^{\pi}(T)\right]\right\} .
$$

In addition, according to Chang et al. [26], optimal control problem with delay is generally an infinite-dimensional problem. To obtain the optimal explicit solution, some additional conditions will be attached. We assume that the value function $V(\cdot)$ is only related to $x, l, v$, and $z$. However, $L^{\pi}(t)$ is related to $M^{\pi}(t)$. In order to make $V(\cdot)$ only depend on $(t, x, l, v, z)$ and then the problem can obtain the optimal explicit expression, we suppose that the following conditions hold:

$$
\begin{aligned}
\gamma_{2} & =\beta e^{-A h}, \\
\bar{\gamma}_{1}-A \beta & =\left(r-\gamma_{1}-\gamma_{2}+\beta\right) \beta .
\end{aligned}
$$

Remark 1. Note that the two assumptions in (19) will be applied over and over below. It ensures that when Elsanosi et al. [22] and Shen and Zeng [28] use the maximum principle to seek explicit expression, the third adjoint equation has zero solution, which is one of the sufficient conditions for the optimality of the control problem with the delay. It is also one of the sufficient conditions for finding explicit solutions of stochastic control problems with delay by using the dynamic programming principle (19). It can be explained as follows: firstly, the insurer calculates the integrated delayed wealth $L(t)$ and the pointwise delayed wealth $M(t)$ at the time $t \in[0, T]$ by selecting the average parameter $A$ and the delay parameter $h$. Then, the insurer selects the parameter $\beta$ to determine the weight between $X(t)$ and $L(t)$ in the mean-variance performance measure. Finally, the insurer sets parameters $\gamma_{2}=\beta e^{-A h}$ and $\gamma_{1}=\left(\beta \int_{-h}^{0} e^{A u} \mathrm{~d} u / 1+\beta \int_{-h}^{0} e^{A u} \mathrm{~d} u\right)\left(r-\gamma_{2}+\beta+A\right)$ according to (19) to determine the weight proportion of past performance $X(t)-\bar{L}(t)$ and $X(t)-M(t)$ at the time $t \in[0, T]$ and adjusts the inflow/outflow of capital accordingly.

Due to the fact that there exists a nonlinear function of the expectation of terminal wealth in the objective functional (18), the optimization problem is time-inconsistent. Most literature solves the mean-variance problem by setting a precommitment, in which optimal strategies obtained are time-inconsistent. In fact, time-consistency cannot be ignored for a rational decision-maker who aims to seek an equilibrium strategy that is optimal at a time and remains optimal as time goes forward into the future. Hence, we shall define the following time-consistent equilibrium strategy according to Bjork and Murgoci [11] and Björk et al. [12].

Definition 2. Consider an admissible strategy $\pi^{*}$, which can be informally viewed as a candidate equilibrium strategy. Choose arbitrarily a fixed $\hat{\pi} \in \Pi$, a real number $\varepsilon(>0)$, and a given initial point $(t, x, l, v, z) \in[0, T] \times \mathbb{R} \times \mathbb{R} \times \mathbb{R} \times\{0,1\}$; define the strategy $\pi_{\varepsilon}$ as follows:

$$
\pi_{\varepsilon}= \begin{cases}\widehat{\pi}(u, x, l, v, z), & (u, x, l, v, z) \in[t, t+\varepsilon) \times \mathbb{R} \times \mathbb{R} \times \mathbb{R} \times\{0,1\}, \\ \pi^{*}(u, x, l, v, z), & (u, x, l, v, z) \in[t+\varepsilon, T] \times \mathbb{R} \times \mathbb{R} \times \mathbb{R} \times\{0,1\}\end{cases}
$$

If

$$
\lim _{\varepsilon \downarrow 0} \inf \frac{J\left(t, x, l, v, z ; \pi^{*}\right)-J\left(t, x, l, v, z ; \pi_{\varepsilon}\right)}{\varepsilon} \geq 0,
$$

we call that $\pi^{*}$ is an equilibrium strategy and the equilibrium value function is given by

$$
\begin{aligned}
J\left(t, x, l, v, z ; \pi^{*}\right)= & E_{t, x, l, v, z}\left[X^{\pi^{*}}(T)+\beta L^{\pi^{*}}(T)\right] \\
& -\frac{\omega}{2} \operatorname{Var}_{t, x, l, v, z}\left[X^{\pi^{*}}(T)+\beta L^{\pi^{*}}(T)\right] .
\end{aligned}
$$

According to Definition 2, the equilibrium strategy above is time-consistent. We aim to seek an equilibrium 
strategy $\pi^{*}$ and the corresponding to equilibrium value function. To give the extended HJB system and Verification Theorem conveniently, we define a variational operator. Let $\mathscr{C}^{1,2,1,2}([0, T] \times \mathbb{R} \times \mathbb{R} \times \mathbb{R} \times\{0,1\})$ denote a space of any function $\varphi(t, x, l, v, z)$ where $\varphi(t, x, l, v, z)$ itself and its derivatives $\varphi_{t}(t, x, l, v, z), \varphi_{x},(t, x, l, v, z), \varphi_{x x}(t, x, l, v, z)$, $\varphi_{x v}(t, x, l, v, z), \quad \varphi_{v}(t, x, l, v, z), \quad \varphi_{v v}(t, x, l, v, z), \quad$ and $\varphi_{l}(t, x, l, v, z)$ are continuous on $[0, T] \times \mathbb{R} \times \mathbb{R} \times \mathbb{R} \times\{0,1\}$. For any function $\varphi(t, x, l, v, z) \in \mathscr{C}^{1,2,1,2}([0, T] \times \mathbb{R} \times \mathbb{R} \times$ $\mathbb{R} \times\{0,1\})$ and a given $\pi \in \Pi$, the variational operator under the CL model is defined as follows:

$$
\begin{aligned}
\mathscr{L}^{\pi} \varphi(t, x, l, v, z)= & \varphi_{t}+\left[\left(r-\gamma_{1}-\gamma_{2}\right) x+\bar{\gamma}_{1} l+\gamma_{2} m+\delta p_{1}(t) v+\left(1+\eta_{1}\right) a_{1} q_{1}(t)\right. \\
& \left.+\left(1+\eta_{2}\right) a_{2} q_{2}(t)+\xi_{1} a_{1}+\xi_{2} a_{2}+\vartheta p_{2}(t)(1-z)\right] \varphi_{x}+\frac{1}{2} p_{2}^{2}(t) v \varphi_{x x} \\
& +\left(x-A l-e^{-A h} m\right) \varphi_{l}+\kappa(\alpha-v) \varphi_{v}+\frac{1}{2} \sigma^{2} v \varphi_{v v}+p_{1}(t) \sigma v \rho \varphi_{x v} \\
& +\lambda_{1} E\left[\varphi\left(t, x-q_{1} Y_{1 i}, l, v, z\right)-\varphi(t, x, l, v, z)\right]+\lambda_{2} E\left[\varphi\left(t, x-q_{2} Y_{2 i}, l, v\right)-\varphi(t, x, l, v, z)\right] \\
& +\lambda E\left[\varphi\left(t, x-q_{1} Y_{1 i}-q_{2} Y_{2 i}, l, v, z\right)-\varphi(t, x, l, v, z)\right] \\
& \left.+\varphi\left(t, x-\zeta p_{2}(t), l, v, z+1\right)-\varphi(t, x, l, v, z)\right] k^{\mathbb{P}}(1-z), \quad z \in\{0,1\} .
\end{aligned}
$$

Before finding the equilibrium strategy, the following theorem gives the verifications for the extended HJB equation corresponding to problem (18).
Theorem 1 (verification theorem). For the mean-variance problem (18), we assume that there exist two real-valued functions $V(t, x, l, v, z)$ and $g(t, x, l, v, z) \in \mathscr{C}^{1,2,1,2}([0, T] \times$ $\mathbb{R} \times \mathbb{R} \times \mathbb{R} \times\{0,1\}$ ) satisfying the following extended $H J B$ equations:

$$
\left\{\begin{array}{l}
\sup _{\pi \in \Pi}\left\{\mathscr{L}^{\pi} V(t, x, l, v, z)-\frac{\omega}{2} \mathscr{L}^{\pi} g^{2}(t, x, l, v, z)+\omega g(t, x, l, v, z) \mathscr{L}^{\pi} g(t, x, l, v, z)\right\}=0, \\
\mathscr{L}^{\pi^{*}} g(t, x, l, v, z)=0, \\
\pi^{*}=\underset{\pi \in \Pi}{\arg \sup }\left\{\mathscr{L}^{\pi} V(t, x, l, v, z)-\frac{\omega}{2} \mathscr{L}^{\pi} g^{2}(t, x, l, v, z)+\omega g(t, x, l, v, z) \mathscr{L}^{\pi} g(t, x, l, v, z)\right\}, \\
V(T, x, l, v, z)=x+\beta l, \quad g(T, x, l, v, z)=x+\beta l,
\end{array}\right.
$$

and then $E_{t, x, l, v, z}\left[X^{\pi^{*}}(T)+\beta L^{\pi^{*}}(T)\right]=g(t, x, l, v, z)$, $J\left(t, x, l, v, z ; \pi^{*}\right)=V(t, x, l, v, z)$ and $\pi^{*}$ is an equilibrium investment-reinsurance strategy.

Proof. See Appendix A.

\section{Solution to the Optimization}

In this section, we derive the explicit solutions of the equilibrium strategy and equilibrium value function for investment-reinsurance problem (18) with delay and 
common shock dependence for postdefault case $(z=1)$ and predefault case $(z=0)$, respectively.
For the postdefault case $(z=1)$, we have $p(t, \bar{T})=0$ and then $p_{2}(t)=0$ for $t \in[\tau, T]$. Hence, according to the variational operator, (23) and (24) can be rewritten as

$$
\begin{aligned}
& \sup _{\pi \in \Pi}\left\{V_{t}+\left[\left(r-\gamma_{1}-\gamma_{2}\right) x+\bar{\gamma}_{1} l+\gamma_{2} m+\delta p_{1} v+\left(1+\eta_{1}\right) a_{1} q_{1}+\left(1+\eta_{2}\right) a_{2} q_{2}+\xi_{1} a_{1}\right.\right. \\
& \left.+\xi_{2} a_{2}\right] V_{x}+\left(x-A l-e^{-A h} m\right) V_{l}+\kappa(\alpha-v) V_{v}+\frac{1}{2} p_{1}^{2} v\left(V_{x x}-\omega g_{x}^{2}\right)+\frac{1}{2} \sigma^{2} v\left(V_{v v}-\omega g_{v}^{2}\right) \\
& +p_{1} \sigma v \rho\left(V_{x v}-\omega g_{v} g_{x}\right)+\lambda_{1}\left[E\left[V\left(t, x-q_{1} Y_{1 i}, l, v, 1\right)-V(t, x, l, v, 1)\right]\right. \\
& \left.-\frac{\omega}{2} E\left[g^{2}\left(t, x-q_{1} Y_{1 i}, l, v, 1\right)-g^{2}(t, x, l, v, 1)\right]+\omega g E\left[g\left(t, x-q_{1} Y_{1 i}, l, v, 1\right)-g(t, x, l, v, 1)\right]\right] \\
& +\lambda_{2}\left[E\left[V\left(t, x-q_{2} Y_{2 i}, l, v, 1\right)-V(t, x, l, v, 1)\right]-\frac{\omega}{2} E\left[g^{2}\left(t, x-q_{2} Y_{2 i}, l, v, 1\right)-g^{2}(t, x, l, v, 1)\right]\right. \\
& \left.+\omega g E\left[g\left(t, x-q_{2} Y_{2 i}, l, v, 1\right)-g(t, x, l, v, 1)\right]\right]+\lambda\left[E\left[V\left(t, x-q_{1} Y_{1 i}-q_{2} Y_{2 i}, l, v, 1\right)-V(t, x, l, v, 1)\right]\right. \\
& -\frac{\omega}{2} E\left[g^{2}\left(t, x-q_{1} Y_{1 i}-q_{2} Y_{2 i}, l, v, 1\right)-g^{2}(t, x, l, v, 1)\right] \\
& \left.\left.+\omega g E\left[g\left(t, x-q_{1} Y_{1 i}-q_{2} Y_{2 i}, l, v, 1\right)-g(t, x, l, v, 1)\right]\right]\right\}=0, \\
& g_{t}+\left[\left(r-\gamma_{1}-\gamma_{2}\right) x+\bar{\gamma}_{1} l+\gamma_{2} m+\delta p_{1}^{*} v+\left(1+\eta_{1}\right) a_{1} q_{1}^{*}+\left(1+\eta_{2}\right) a_{2} q_{2}^{*}+\xi_{1} a_{1}+\xi_{2} a_{2}\right] g_{x} \\
& +\left(x-A l-e^{-A h} m\right) g_{l}+\kappa(\alpha-v) g_{v}+\frac{1}{2} p_{1}^{* 2} v g_{x x}+\frac{1}{2} \sigma^{2} v g_{v v}+p_{1}^{*} \sigma v \rho g_{x v} \\
& +\lambda_{1} E\left[g\left(t, x-q_{1}^{*} Y_{1 i}, l, v, 1\right)-g(t, x, l, v, 1)\right]+\lambda_{2} E\left[g\left(t, x-q_{2}^{*} Y_{2 i}, l, v, 1\right)-g(t, x, l, v, 1)\right] \\
& +\lambda E\left[g\left(t, x-q_{1}^{*} Y_{1 i}-q_{2}^{*} Y_{2 i}, l, v, 1\right)-g(t, x, l, v, 1)\right]=0
\end{aligned}
$$

where the terminal value conditions are given by $V(T, x, l, v, 1)=x+\beta l$ and $g(T, x, l, v, 1)=x+\beta l$. For the predefault case $(z=0)$, according to (23) and (24), we derive 


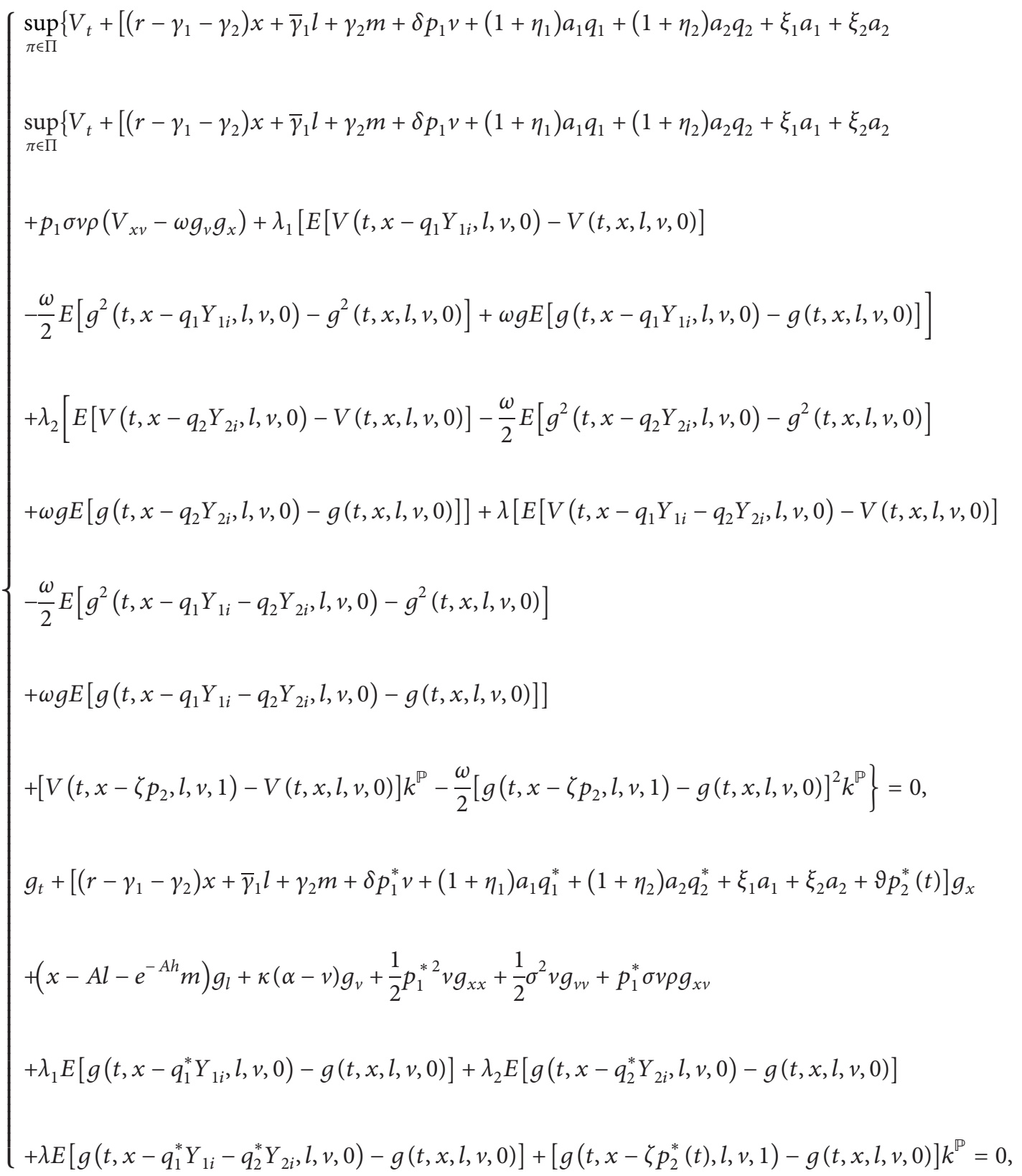

where the terminal value conditions are given by $V(T, x, l, v, 0)=x+\beta l$ and $g(T, x, l, v, 0)=x+\beta l$. Next, we give the explicit solution of the equilibrium strategies and the corresponding equilibrium value function by the following theorem. Noting that $q_{1}(\cdot)$ and $q_{2}(\cdot)$ are nonnegative, the following three cases must be discussed:

Case 1: for $\eta_{1} \leq\left(\lambda \mu_{11} \mu_{21} / b_{2}^{2}\right)\left(a_{2} / a_{1}\right) \eta_{2}$, we have $\left(a_{1} \eta_{1} b_{2}^{2}-a_{2} \eta_{2} \lambda \mu_{11} \mu_{21} / b_{1}^{2} b_{2}^{2}-\lambda^{2} \mu_{11}^{2} \mu_{21}^{2}\right) \leq 0$ and $\left(a_{2} \eta_{2}\right.$ $\left.b_{1}^{2}-a_{1} \eta_{1} \lambda \mu_{11} \mu_{21} / b_{1}^{2} b_{2}^{2}-\lambda^{2} \mu_{11}^{2} \mu_{21}^{2}\right)<0$.
Case 2: for $\left(\lambda \mu_{11} \mu_{21} / b_{2}^{2}\right)\left(a_{2} / a_{1}\right) \eta_{2}<\eta_{1}<\left(b_{1}^{2} / \lambda \mu_{11} \mu_{21}\right)$ $\left(a_{2} / a_{1}\right) \eta_{2}$, we have $\left(a_{1} \eta_{1} b_{2}^{2}-a_{2} \eta_{2} \lambda \mu_{11} \mu_{21} / b_{1}^{2} b_{2}^{2}-\right.$ $\left.\lambda^{2} \mu_{11}^{2} \mu_{21}^{2}\right)>0$ and $\left(a_{2} \eta_{2} b_{1}^{2}-a_{1} \eta_{1} \lambda \mu_{11} \mu_{21} / b_{1}^{2} b_{2}^{2}-\lambda^{2} \mu_{11}^{2}\right.$ $\left.\mu_{21}^{2}\right)>0$.

Case 3: for $\eta_{1} \geq\left(b_{1}^{2} / \lambda \mu_{11} \mu_{21}\right)\left(a_{2} / a_{1}\right) \eta_{2}$, we have $\left(a_{1} \eta_{1} b_{2}^{2}-a_{2} \eta_{2} \lambda \mu_{11} \mu_{21} / b_{1}^{2} b_{2}^{2}-\lambda^{2} \mu_{11}^{2} \mu_{21}^{2}\right)>0$ and $\left(a_{2} \eta_{2}\right.$ $\left.b_{1}^{2}-a_{1} \eta_{1} \lambda \mu_{11} \mu_{21} / b_{1}^{2} b_{2}^{2}-\lambda^{2} \mu_{11}^{2} \mu_{21}^{2}\right) \leq 0$.

Theorem 2. For the mean-variance problem (18) under Case 2 , the equilibrium investment and reinsurance strategies are given by 


$$
\begin{aligned}
& p_{1}^{*}(t)=\left\{\begin{array}{l}
\frac{\delta}{\omega} e^{-\left(r-\gamma_{1}-\gamma_{2}+\beta\right)(T-t)}-\frac{\delta^{2} \sigma \rho}{\omega(\kappa+\delta \sigma \rho)}\left[1-e^{-(\kappa+\delta \sigma \rho)(T-t)}\right] e^{-\left(r-\gamma_{1}-\gamma_{2}+\beta\right)(T-t)}, \\
\kappa+\delta \sigma \rho \neq 0, \\
\frac{\delta}{\omega} e^{-\left(r-\gamma_{1}-\gamma_{2}+\beta\right)(T-t)}-\frac{\delta^{2} \sigma \rho}{\omega}(T-t) e^{-\left(r-\gamma_{1}-\gamma_{2}+\beta\right)(T-t)}, \quad \kappa+\delta \sigma \rho=0,
\end{array}\right. \\
& p_{2}^{*}(t)=\left[\frac{\vartheta}{\omega \zeta^{2} k^{\mathbb{P}}}+\frac{1}{\omega \zeta}\left(R(t)-R_{0}(t)\right)-\frac{1}{\omega \zeta}\right] e^{-\left(r-\gamma_{1}-\gamma_{2}+\beta\right)(T-t)} \mathbf{1}_{\tau>t}, \\
& q_{1}^{*}(t)=\frac{a_{1} \eta_{1} b_{2}^{2}-a_{2} \eta_{2} \lambda \mu_{11} \mu_{21}}{b_{1}^{2} b_{2}^{2}-\lambda^{2} \mu_{11}^{2} \mu_{21}^{2}} \frac{1}{\omega} e^{-\left(r-\gamma_{1}-\gamma_{2}+\beta\right)(T-t)}, \\
& q_{2}^{*}(t)=\frac{a_{2} \eta_{2} b_{1}^{2}-a_{1} \eta_{1} \lambda \mu_{11} \mu_{21}}{b_{1}^{2} b_{2}^{2}-\lambda^{2} \mu_{11}^{2} \mu_{21}^{2}} \frac{1}{\omega} e^{-\left(r-\gamma_{1}-\gamma_{2}+\beta\right)(T-t)},
\end{aligned}
$$

and the equilibrium value function is

$$
V(t, x, l, v, z)=\left\{\begin{array}{l}
e^{\left(r-\gamma_{1}-\gamma_{2}+\beta\right)(T-t)}(x+\beta l)+\frac{F(t)}{\omega} v+\frac{G(t)}{\omega}, \quad z=1, \\
e^{\left(r-\gamma_{1}-\gamma_{2}+\beta\right)(T-t)}(x+\beta l)+\frac{F_{0}(t)}{\omega} v+\frac{G_{0}(t)}{\omega}, \quad z=0 .
\end{array}\right.
$$

Besides, the expectation and variance of the terminal value corresponding to the equilibrium strategies are given by

$$
\begin{gathered}
E_{t, x, l, v, z}\left[X^{\pi^{*}}(T)+\beta L^{\pi^{*}}(T)\right]=\left\{\begin{array}{l}
e^{\left(r-\gamma_{1}-\gamma_{2}+\beta\right)(T-t)}(x+\beta l)+\frac{Q(t)}{\omega} v+\frac{R(t)}{\omega}, \quad z=1, \\
e^{\left(r-\gamma_{1}-\gamma_{2}+\beta\right)(T-t)}(x+\beta l)+\frac{Q_{0}(t)}{\omega} v+\frac{R_{0}(t)}{\omega}, \quad z=0,
\end{array}\right. \\
\operatorname{Var}_{t, x, l, v, z}\left[X^{\pi^{*}}(T)+\beta L^{\pi^{*}}(T)\right]=\left\{\begin{array}{l}
\frac{2}{\omega^{2}}[(Q(t)-F(t)) v+(R(t)-G(t))], \quad z=1, \\
\frac{2}{\omega^{2}}\left[\left(Q_{0}(t)-F_{0}(t)\right) v+\left(R_{0}(t)-G_{0}(t)\right)\right], \quad z=0,
\end{array}\right.
\end{gathered}
$$

where $Q(t), F(t), R(t)$, and $G(t)$ are given by (B.19)-(B.22), and $Q_{0}(t), F_{0}(t), R_{0}(t)$, and $G_{(t)}$ are given by $(B .38)-(B .41)$, respectively.

Proof. See Appendix B.

Remark 2.

(i) For Case 1, we have $q_{1}(t)=0$, and then we just need to calculate $q_{2}(t)$, and the calculation process is similar to Theorem 2. Parallelly, for Case 3, it is easy to obtain $q_{2}(t)=0$, and only $q_{1}(t)$ remains to be calculated.

(ii) It is easy to see from Theorem 2 that both equilibrium investment and proportional reinsurance strategies are deterministic functions and they are independent of the wealth process $X(t)$ and the volatility process $v(t)$ of risky assets. From Theorem 2, equilibrium strategies are not directly affected by $A$ and $h$, but, according to Remark 1, $A$ and $h$ have indirect effects on equilibrium strategies. 
(iii) From Theorem 2, we can also find that the equilibrium investment strategy with delay under Heston's SV model can be decomposed into two parts. The first part $(\delta / \omega) e^{-\left(r-\gamma_{1}-\gamma_{2}+\beta\right)(T-t)}$ is related to the appreciation rate of the risky asset mainly. The

$-\left(\delta^{2} \sigma \rho / \omega(\kappa+\delta \sigma \rho)\right)[1-$

second part $\left.e^{-(\kappa+\delta \sigma \rho)(T-t)}\right] e^{-\left(r-\gamma_{1}-\gamma_{2}+\beta\right)(T-t)}$ is called a modification factor by us and demonstrates the effect resulting from the stochastic volatility under Heston's SV model.

(iv) According to (22), we have

$$
\begin{aligned}
& \operatorname{Var}_{t, x, l, v, z}\left[X^{\pi^{*}}(T)+\beta L^{\pi^{*}}(T)\right] \\
& \quad=(2 / \omega)\left(E_{t, x, l, v, z}\left[X^{\pi^{*}}(T)+\beta L^{\pi^{*}}(T)\right]-J\left(t, x, l, v, z ; \pi^{*}\right)\right),
\end{aligned}
$$

From (30), it is easy to obtain that

$$
\frac{1}{\omega}=\left\{\begin{array}{l}
\sqrt{\frac{\operatorname{Var}_{t, x, l, v, z}\left[X^{\pi^{*}}(T)+\beta L^{\pi^{*}}(T)\right]}{2[(Q(t)-F(t)) v+(R(t)-G(t))]}}, \quad z=1, \\
\sqrt{\frac{\operatorname{Var}_{t, x, l, v, z}\left[X^{\pi^{*}}(T)+\beta L^{\pi^{*}}\right.}{2\left[\left(Q_{0}(t)-F_{0}(t)\right) v+\left(R_{0}(t)-G_{0}(t)\right)\right]}}, \quad z=0 .
\end{array}\right.
$$

Together with equation (29), we derive

$$
E_{t, x, l, v, z}\left[X^{\pi^{*}}(T)+\beta L^{\pi^{*}}(T)\right]=\left\{\begin{array}{l}
e^{\left(r-\gamma_{1}-\gamma_{2}+\beta\right)(T-t)}(x+\beta l) \\
+\sqrt{\frac{\operatorname{Var}_{t, x, l, v, z}\left[X^{\pi^{*}}(T)+\beta L^{\pi^{*}}(T)\right](Q(t) v+R(t))^{2}}{2[(Q(t)-F(t)) v+(R(t)-G(t))]},} z=1, \\
e^{\left(r-\gamma_{1}-\gamma_{2}+\beta\right)(T-t)}(x+\beta l) \\
+\sqrt{\frac{\operatorname{Var}_{t, x, l, v, z}\left[X^{\pi^{*}}(T)+\beta L^{\pi^{*}}(T)\right]\left(Q_{0}(t) v+R_{0}(t)\right)^{2}}{2\left[\left(Q_{0}(t)-F_{0}(t)\right) v+\left(R_{0}(t)-G_{0}(t)\right)\right]},} z=0 .
\end{array}\right.
$$

Equation (33) is known as the efficient frontier of the investment-reinsurance problem with delay under Heston's SV model at the initial state $(t, x, l, v, z)$ in modern portfolio theory. The efficient frontier is also a straight line in the mean-standard deviation plane, no matter at which state.
From Theorem 2, we can present the following corollary without considering the defaultable bond.

Corollary 1 (no defaultable bond case). With no defaultable bond, the optimal time-consistent strategies under Case 2 are given by

$$
\begin{aligned}
& p_{1}^{*}(t)=\left\{\begin{array}{l}
\frac{\delta}{\omega} e^{-\left(r-\gamma_{1}-\gamma_{2}+\beta\right)(T-t)}-\frac{\delta^{2} \sigma \rho}{\omega(\kappa+\delta \sigma \rho)}\left[1-e^{-(\kappa+\delta \sigma \rho)(T-t)}\right] e^{-\left(r-\gamma_{1}-\gamma_{2}+\beta\right)(T-t)}, \\
\kappa+\delta \sigma \rho \neq 0, \\
\frac{\delta}{\omega} e^{-\left(r-\gamma_{1}-\gamma_{2}+\beta\right)(T-t)}-\frac{\delta^{2} \sigma \rho}{\omega}(T-t) e^{-\left(r-\gamma_{1}-\gamma_{2}+\beta\right)(T-t)}, \quad \kappa+\delta \sigma \rho=0,
\end{array}\right. \\
& q_{1}^{*}(t)=\frac{a_{1} \eta_{1} b_{2}^{2}-a_{2} \eta_{2} \lambda \mu_{11} \mu_{21}}{b_{1}^{2} b_{2}^{2}-\lambda^{2} \mu_{11}^{2} \mu_{21}^{2}} \frac{1}{\omega} e^{-\left(r-\gamma_{1}-\gamma_{2}+\beta\right)(T-t)}, \\
& q_{2}^{*}(t)=\frac{a_{2} \eta_{2} b_{1}^{2}-a_{1} \eta_{1} \lambda \mu_{11} \mu_{21}}{b_{1}^{2} b_{2}^{2}-\lambda^{2} \mu_{11}^{2} \mu_{21}^{2}} \frac{1}{\omega} e^{-\left(r-\gamma_{1}-\gamma_{2}+\beta\right)(T-t)},
\end{aligned}
$$


and the corresponding value function is as follows:

$$
V(t, x, l, v)=e^{\left(r-\gamma_{1}-\gamma_{2}+\beta\right)(T-t)}(x+\beta l)+\frac{F(t)}{\omega} v+\frac{G(t)}{\omega},
$$

where $F(t)$ and $G(t)$ are given by (B.20) and (B.22), respectively.

If $A=\gamma_{1}=\gamma_{2}=h=0$, then the model in this paper degenerates as the special cases without delay. Furthermore, according to (19), we have also $\beta=0$ and $\bar{\gamma}_{1}=0$ for $A=\gamma_{1}=\gamma_{2}=h=0$. Hence, by Theorem 2, we give the following corollary.

Corollary 2. Without delay case, the optimal time-consistent investment and reinsurance strategies under Case 2 are given by

$$
\begin{aligned}
p_{1}^{*}(t) & =\left\{\begin{array}{l}
\frac{\delta}{\omega} e^{-r(T-t)}-\frac{\delta^{2} \sigma \rho}{\omega(\kappa+\delta \sigma \rho)}\left[1-e^{-(\kappa+\delta \sigma \rho)(T-t)}\right] e^{-r(T-t)}, \quad \kappa+\delta \sigma \rho \neq 0, \\
\frac{\delta}{\omega} e^{-r(T-t)}-\frac{\delta^{2} \sigma \rho}{\omega}(T-t) e^{-r(T-t)}, \quad \kappa+\delta \sigma \rho=0,
\end{array}\right. \\
= & \left\{\begin{array}{l}
\frac{\kappa+\delta \rho \sigma e^{(\delta \rho \sigma+\kappa)(t-T)}}{\omega(\delta \rho \sigma+\kappa)} \delta e^{r(t-T)}, \quad \kappa+\delta \sigma \rho \neq 0, \\
\frac{\delta}{\omega}[1+\delta \sigma \rho(t-T)] e^{r(t-T)}, \quad \kappa+\delta \sigma \rho=0, \\
p_{2}^{*}(t)= \\
q_{2}^{*}(t)= \\
\frac{\vartheta}{\left.\omega \zeta^{2} k^{\mathbb{P}}+\frac{1}{\omega \zeta}\left(\widetilde{R}(t)-\widetilde{R}_{0}(t)\right)-\frac{1}{\omega \zeta}\right] e^{-r(T-t)} \mathbf{1}_{\tau>t},} \\
q_{1}^{*}(t)=\frac{a_{1} \eta_{1} b_{2}^{2}-a_{2} \eta_{2} \lambda \mu_{11} \mu_{21}}{b_{1}^{2} b_{2}^{2}-\lambda_{1}^{2} \mu_{11}^{2} \mu_{21}^{2}} \frac{1}{\omega} e^{-r(T-t)}, \lambda_{1}^{2} \eta_{1} \lambda \mu_{11} \mu_{21}^{2} \mu_{11}^{2} \frac{1}{\omega} e^{-r(T-t)},
\end{array}\right.
\end{aligned}
$$

and the corresponding equilibrium value function is

$$
V(t, x, l, v, z)=\left\{\begin{array}{l}
e^{r(T-t)} x+\frac{F(t)}{\omega} v+\frac{\widetilde{G}(t)}{\omega}, \quad z=1, \\
e^{r(T-t)} x+\frac{F_{0}(t)}{\omega} v+\frac{\widetilde{G}_{0}(t)}{\omega}, \quad z=0,
\end{array}\right.
$$




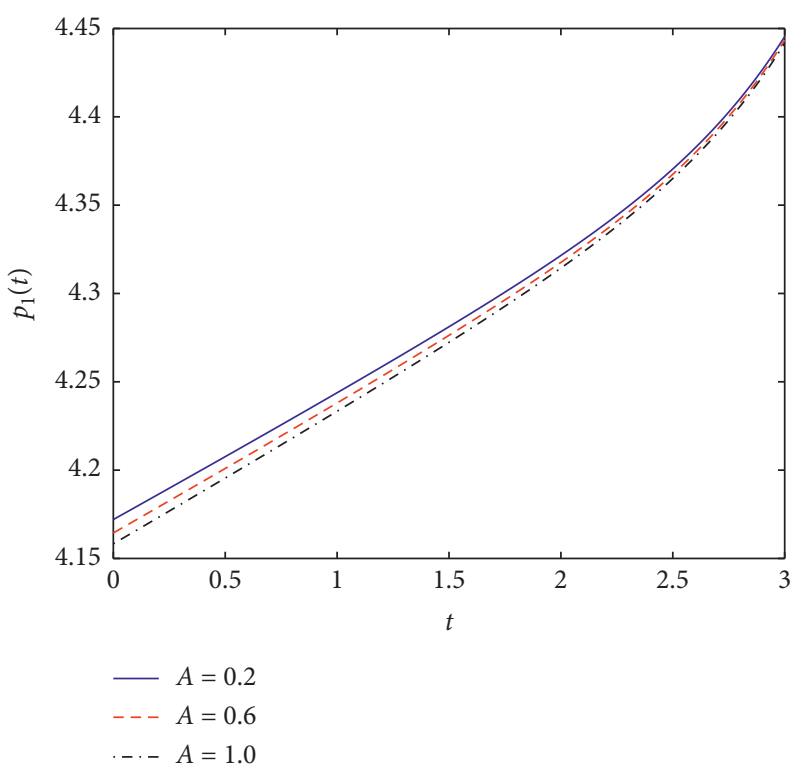

Figure 1: The effect of $A$ on $p_{1}(t)$.

where

$$
\begin{aligned}
\widetilde{R}(t)= & \frac{\omega\left(\xi_{1} a_{1}+\xi_{2} a_{2}\right)}{r}\left[e^{r(T-t)}-1\right]+\left(a_{1} \eta_{1} n_{1}+a_{2} \eta_{2} n_{2}\right)(T-t)+\kappa \alpha \int_{t}^{T} Q(u) \mathrm{d} u, \\
\widetilde{R}_{0}(t)= & e^{(\vartheta / \zeta)} \int_{t}^{T} e^{-(\vartheta / \zeta)}\left[\frac{\vartheta}{\zeta} \widetilde{R}(u)+\omega\left(\xi_{1} a_{1}+\xi_{2} a_{2}\right) e^{r(T-u)}+\kappa \alpha Q_{0}(u)+a_{1} \eta_{1} n_{1}+a_{2} \eta_{2} n_{2}\right. \\
& \left.+\frac{\vartheta^{2}}{\zeta^{2} k^{\mathbb{P}}}-\frac{2 \vartheta}{\zeta}+k^{\mathbb{P}}\right] \mathrm{d} u, \\
\widetilde{G}(t)= & \frac{\omega\left(\xi_{1} a_{1}+\xi_{2} a_{2}\right)}{r}\left[e^{r(T-t)}-1\right]+\left(a_{1} \eta_{1} n_{1}+a_{2} \eta_{2} n_{2}-\frac{1}{2} b_{1}^{2} n_{1}^{2}-\frac{1}{2} b_{2}^{2} n_{2}^{2}\right. \\
& \left.-\lambda \mu_{11} \mu_{21} n_{1} n_{2}\right)(T-t)+\kappa \alpha \int_{t}^{T} F(u) \mathrm{d} u, \\
\widetilde{G}_{0}(t)= & e^{k^{\mathbb{P}} t} \int_{t}^{T} e^{-k^{\mathbb{P}} u}\left[k^{\mathbb{P}} \widetilde{G}(u)+\omega\left(\xi_{1} a_{1}+\xi_{2} a_{2}\right) e^{r(T-u)}+\kappa \alpha F_{0}(u)+a_{1} \eta_{1} n_{1}+a_{2} \eta_{2} n_{2}\right. \\
& \left.-\frac{1}{2} b_{1}^{2} n_{1}^{2}-\frac{1}{2} b_{2}^{2} n_{2}^{2}-\lambda \mu_{11} \mu_{21}+\left(\frac{\vartheta}{\zeta}-k^{\mathbb{P}}\right)\left(\widetilde{R}(t)-\widetilde{R}_{0}(t)\right)+\frac{\vartheta^{2}}{2 k^{\mathbb{P}} \zeta}-\frac{\vartheta}{\zeta}+\frac{k^{\mathbb{P}}}{2}\right] \mathrm{d} u .
\end{aligned}
$$

Remark 3. From Corollaries 1 and 2, under Case 2, we can find that the optimal time-consistent strategies for no delay and no defaultable bond are given by

$$
p_{1}^{*}(t)= \begin{cases}\frac{\kappa+\delta \rho \sigma e^{(\delta \rho \sigma+\kappa)(t-T)}}{\omega(\delta \rho \sigma+\kappa)} \delta e^{r(t-T)}, & \kappa+\delta \sigma \rho \neq 0, \\ \frac{\delta}{\omega}[1+\delta \sigma \rho(t-T)] e^{r(t-T)}, & \kappa+\delta \sigma \rho=0,\end{cases}
$$

$$
\begin{aligned}
& q_{1}^{*}(t)=\frac{a_{1} \eta_{1} b_{2}^{2}-a_{2} \eta_{2} \lambda \mu_{11} \mu_{21}}{b_{1}^{2} b_{2}^{2}-\lambda^{2} \mu_{11}^{2} \mu_{21}^{2}} \frac{1}{\omega} e^{-r(T-t)}, \\
& q_{2}^{*}(t)=\frac{a_{2} \eta_{2} b_{1}^{2}-a_{1} \eta_{1} \lambda \mu_{11} \mu_{21}}{b_{1}^{2} b_{2}^{2}-\lambda^{2} \mu_{11}^{2} \mu_{21}^{2}} \frac{1}{\omega} e^{-r(T-t)} .
\end{aligned}
$$

Further, without considering the risk of common shock dependence, that is, $\lambda=0$, the reinsurance strategies become $q_{1}^{*}(t)=\left(\mu_{11} \eta_{1} / \mu_{12} \omega\right) e^{r(t-T)} \quad$ and $q_{2}^{*}(t)=\left(\mu_{21} \eta_{2} / \mu_{22} \omega\right) e^{r(t-T)}$. After comparison, it is easy to see that the investment strategy in (39) is completely consistent with the result in $\mathrm{Li}$ et al.'s work [14]. But the 


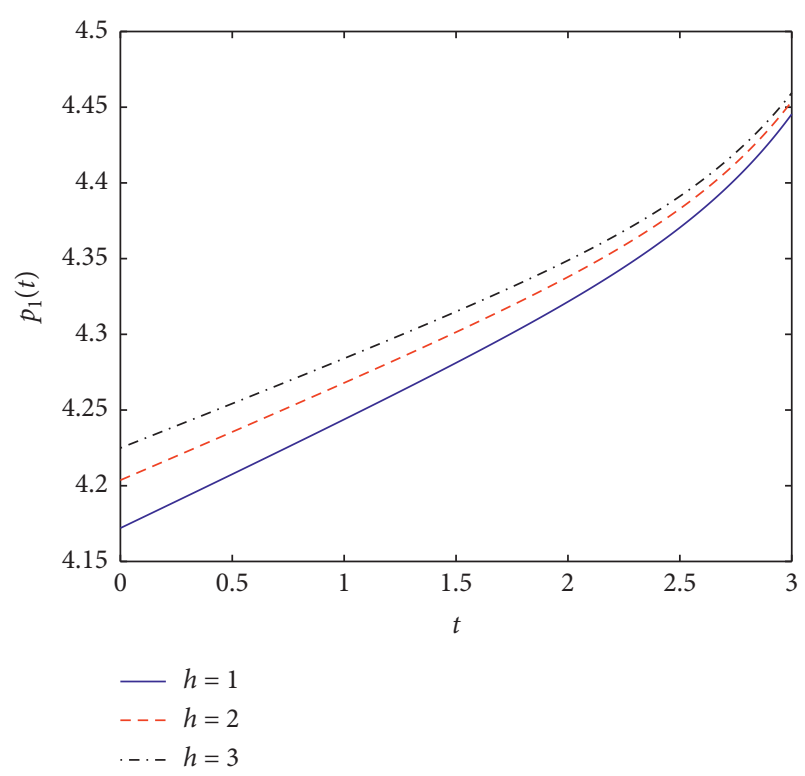

Figure 2: The effect of $h$ on $p_{1}(t)$.

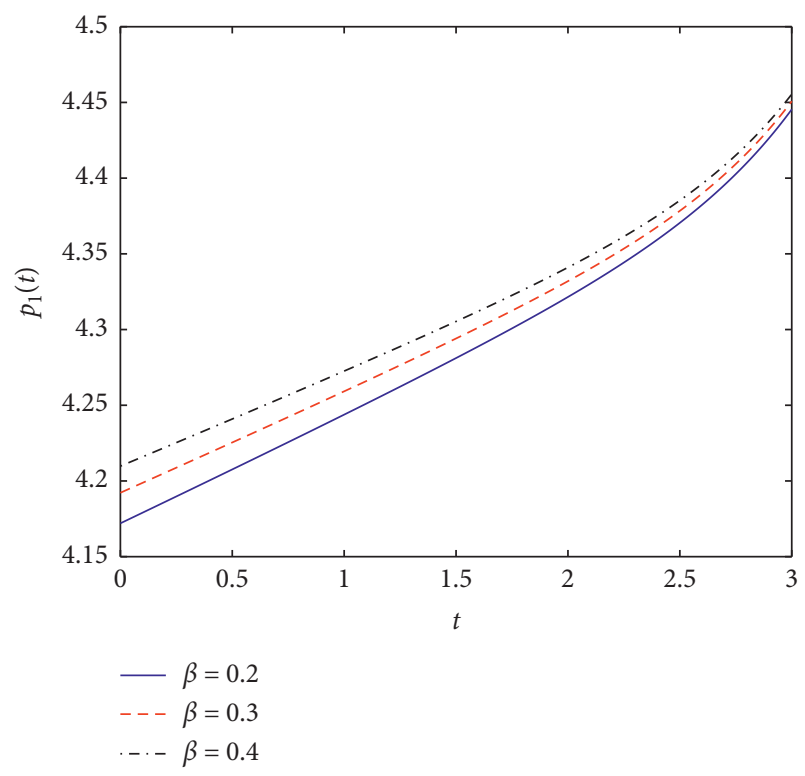

Figure 3: The effect of $\beta$ on $p_{1}(t)$.

reinsurance strategies are slightly different because we employ the expected value principle to calculate the premium, while Li et al. [14] do not.

\section{Sensitivity Analysis}

In this section, we provide numerical simulations to illustrate our results, and the effect of important parameters on equilibrium strategy and economic explanation will be presented. Throughout this section, unless otherwise stated, the basic model parameters are as follows: $\lambda=2, \lambda_{1}=3$, $\lambda_{2}=4, \quad \mu_{11}=\mu_{21}=0.1, \quad \mu_{12}=\mu_{22}=0.2, \quad \theta_{1}=\theta_{2}=0.2$, $\eta_{1}=\eta_{2}=0.6, \quad r=0.02, \quad \sigma=0.8, \quad A=0.2, \quad h=1, \quad \beta=0.2$,

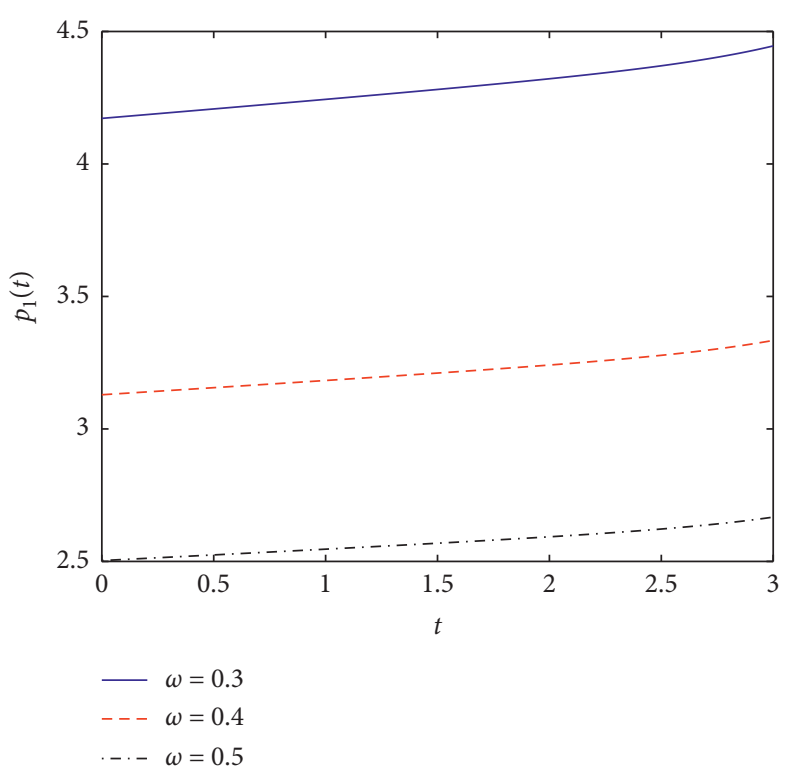

Figure 4: The effect of $\omega$ on $p_{1}(t)$.

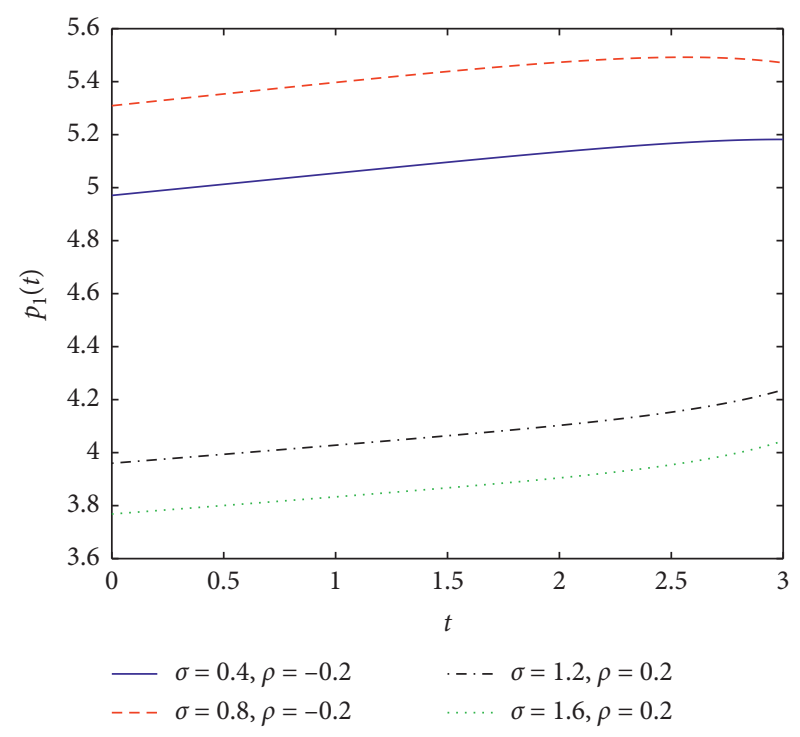

FIgURE 5: The effect of $\sigma$ on $p_{1}(t)$.

$\delta=1.5, \rho=0.2, \kappa=2, \omega=0.3, T=3, \vartheta=0.01, \zeta=0.5$, and $k^{\mathbb{P}}=0.005$. Next, we vary the value of one parameter by fixing other parameters to analyze our results.

Figure 1 describes the effect of parameter $A$ on the equilibrium investment strategy invested in the risky asset, where $A$ takes $0.2,0.6$, and 1 , respectively. The results show that the amount of equilibrium investment decreases with the increase of $A$. According to the definition of average delayed wealth $\bar{L}(t)$, the larger $A$ is, the smaller the proportion of earlier wealth in the construction of average delay wealth is. In other words, the larger $A$ is, the more the insurer pays attention to the wealth close to the current time, which is a kind of short-sighted behavior. In practice, investors' shortsightedness often increases the risk, and they 


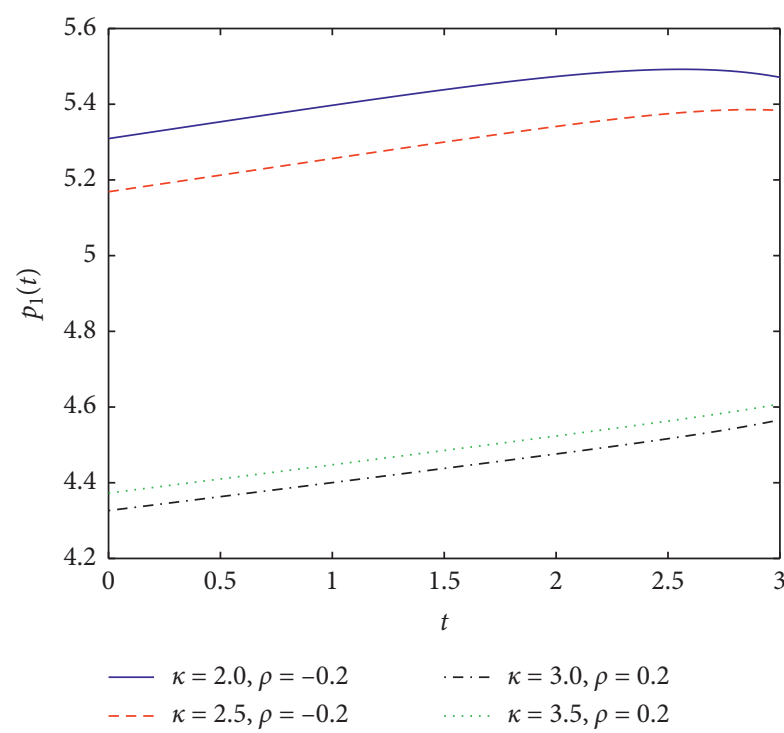

Figure 6: The effect of $\kappa$ on $p_{1}(t)$.

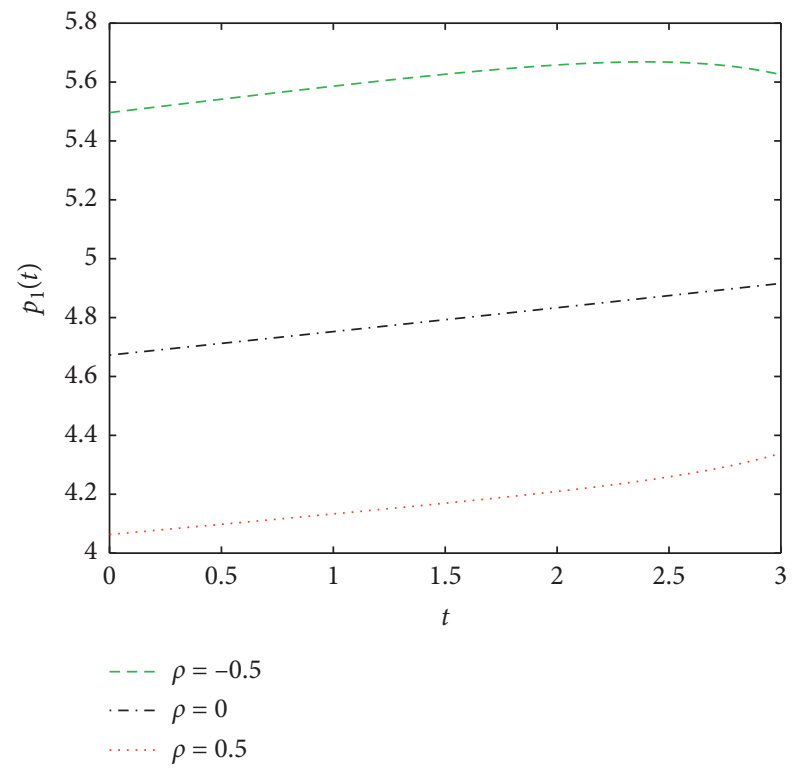

FIgURE 7: The effect of $\rho$ on $p_{1}(t)$.

are likely to take risks for short-term interests. Therefore, when $A$ is large, the insurer will reduce the investment of the risky asset in order to control the overall risk. Figure 2 shows the influence of parameter $h$ on the amount invested in risky assets. It is easy to see that the amount of investment increases with the increase of $h$. Intuitively, with the increase of $h$, the horizontal range of the average becomes longer, and the average delayed wealth is more stable. In other words, with the increase of $h$, the risk faced by the insurer will be reduced, so the amount of the risky asset investment will increase. Figure 3 demonstrates the influence of parameter $\beta$ on the amount invested in risky assets. Obviously, the larger $\beta$ is, the more the equilibrium investment is. In fact, the mechanism of $\beta$ on equilibrium investment is similar to that of $h$. According to the definition of terminal wealth

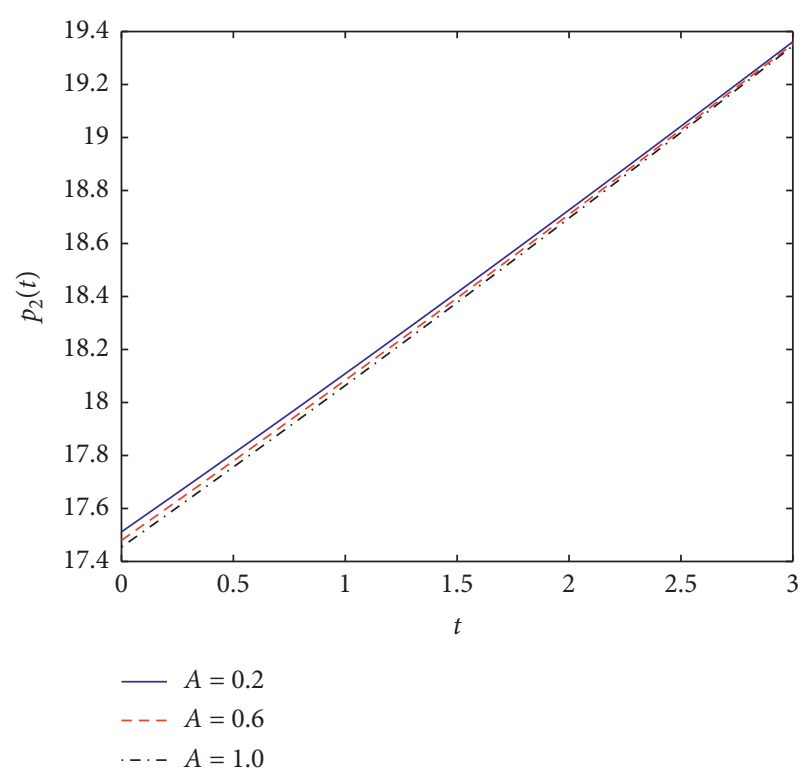

Figure 8: The effect of $A$ on $p_{2}(t)$.

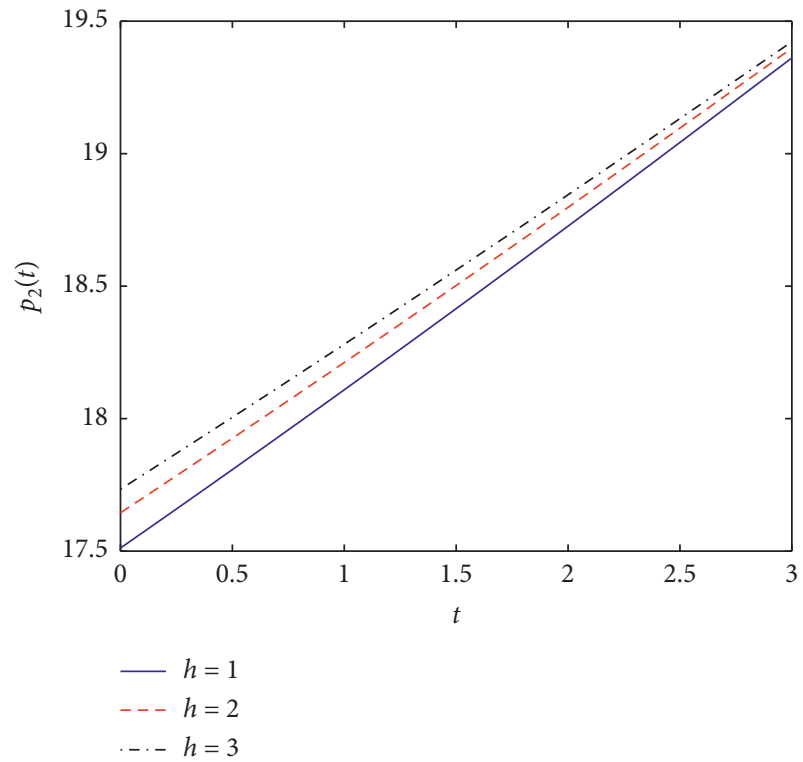

Figure 9: The effect of $h$ on $p_{2}(t)$.

$X(T)+\beta L(T)$, the larger $\beta$ is, the greater the weight of average delayed wealth is in the composition of terminal wealth. In practice, this is a more rational performance of investors. Therefore, with the increase of $\beta$, the risk faced by the insurer is often reduced; thus, the number of the risky assets invested increases. Figure 4 depicts the effect of $\omega$ on the equilibrium investment strategy invested in the risky asset. $\omega$ is the risk aversion coefficient. The larger $\omega$ is, the less amount of the risky asset will be invested, as shown in Figure 4.

Figure 5 depicts the two different effects of the parameter $\sigma$ on the amount invested in the risky asset in two different cases of $\rho>0$ and $\rho<0$. It is easy to find that when $\rho>0$, the amount of equilibrium investment decreases with respect to 


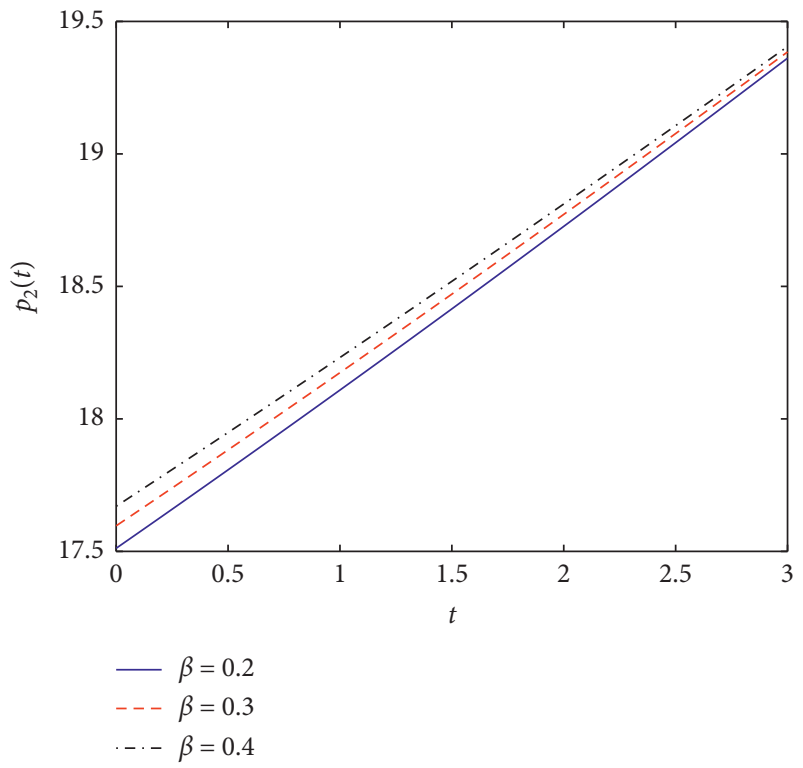

Figure 10: The effect of $\beta$ on $p_{2}(t)$.

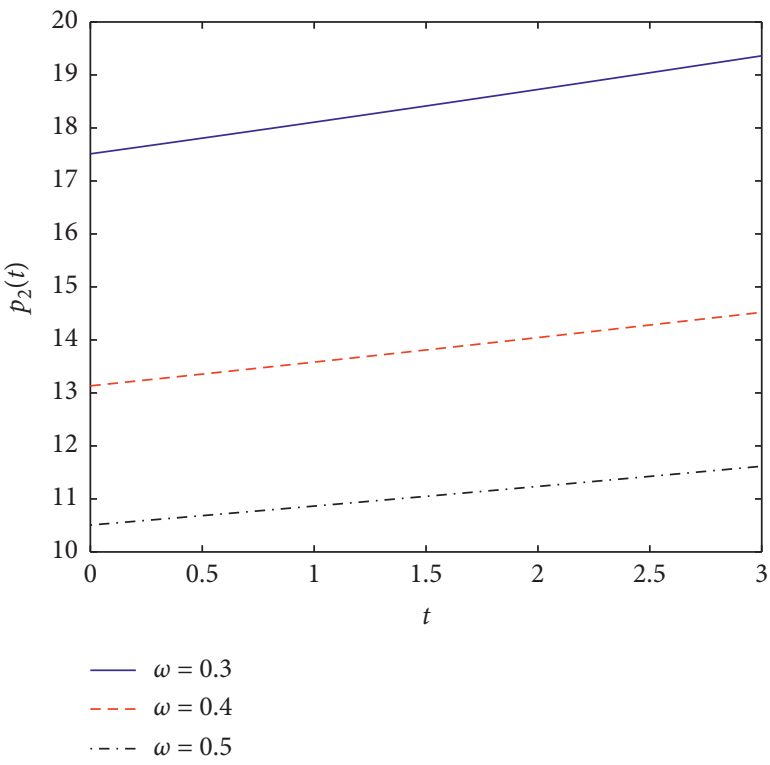

FIgure 11: The effect of $\omega$ on $p_{2}(t)$.

$\sigma$; when $\rho<0$, the amount of equilibrium investment increases with the increase of $\sigma$. This is because when $\rho>0$, the larger $\sigma$ is, the greater the volatility of the risky asset is, so the insurer reduces investment. On the contrary, when $\rho<0$, due to the negative correlation between the price of the risky asset and volatility, with the increase of $\sigma$, the expected price of the risky asset increases, so the investment increases. Figure 6 shows the two different effects of the parameter $\kappa$ on the equilibrium investment strategy invested in the risky asset in two different cases of $\rho>0$ and $\rho<0$. Obviously, when $\rho>0$, the amount of equilibrium investment increases with $\kappa$; when $\rho<0$, the number of equilibrium investment decreases with the increase of $\kappa$. If $\rho>0$, the uncertainties of

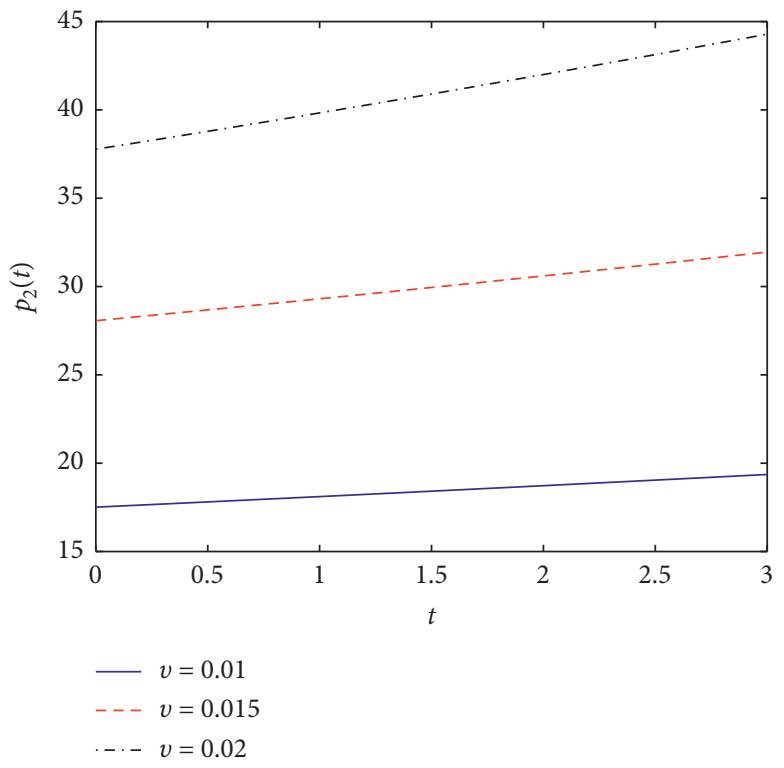

Figure 12: The effect of $\vartheta$ on $p_{2}(t)$.

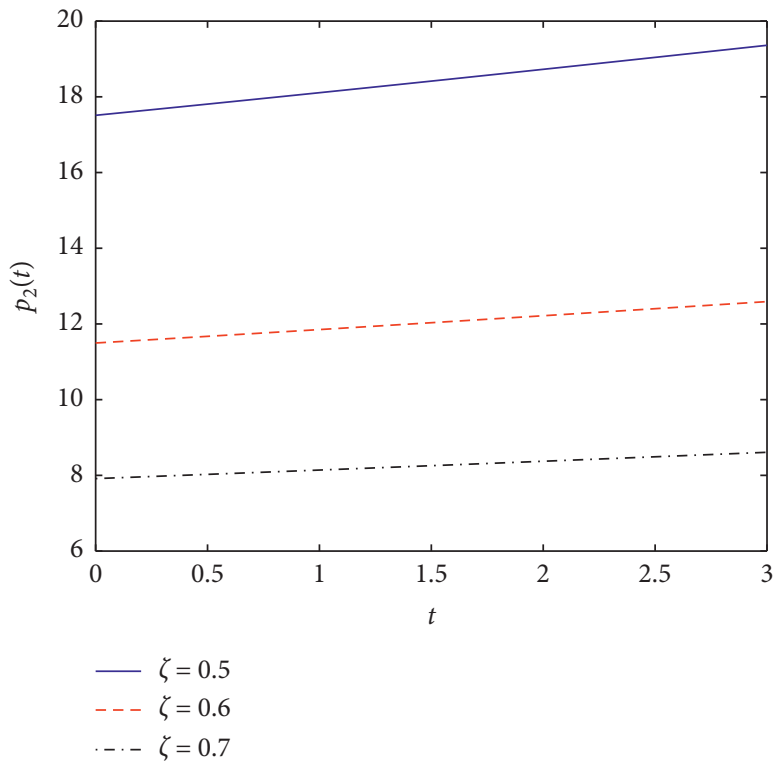

FIgure 13: The effect of $\zeta$ on $p_{2}(t)$.

the risky asset's price and its volatility vary in the same way. $\kappa$ reflects the speed of $v(t)$ toward $\alpha$ when it wanders away. A larger $\kappa$ means more stable volatility of a risky asset, and then the insurer will increase the investment in the risky asset. For $\rho<0$, the uncertainties of $v(t)$ and $S(t)$ vary in different ways. As $\kappa$ increases, $v(t)$ will be more stable. Then, owing to the negative correlation between $v(t)$ and $S(t)$, there is an increased probability of a decrease in the risky asset's price. Thus, the equilibrium investment decreases with $\kappa$ when $\rho<0$. Figure 7 shows the effect of $\rho$ on the amount invested in risky assets. As $\rho$ increases from -1 to 1 , the potential volatility of risky asset prices becomes greater and, therefore, $p_{1}^{*}(t)$ decreases with $\rho$. 


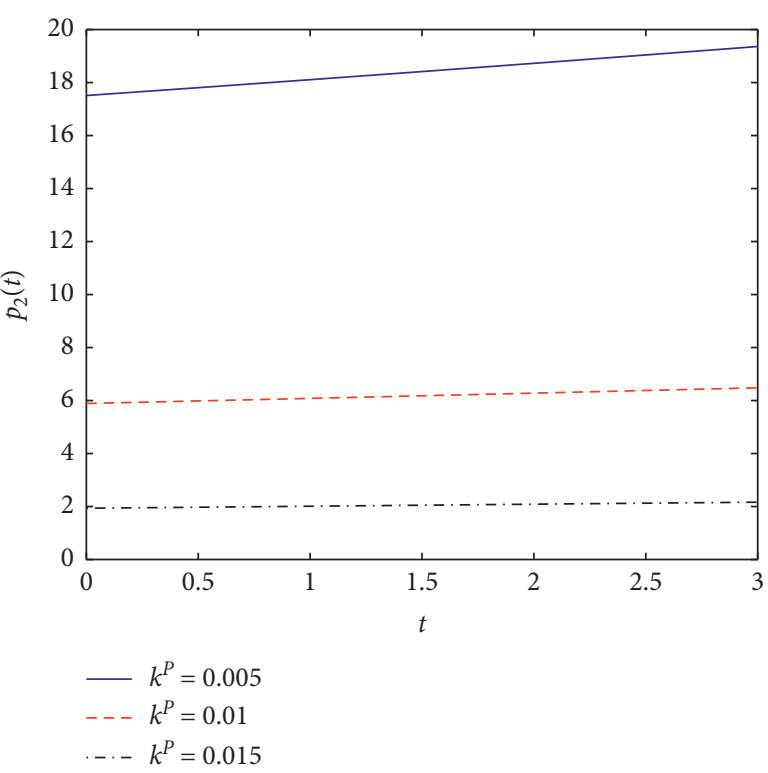

Figure 14: The effect of $k^{\mathbb{P}}$ on $p_{2}(t)$.

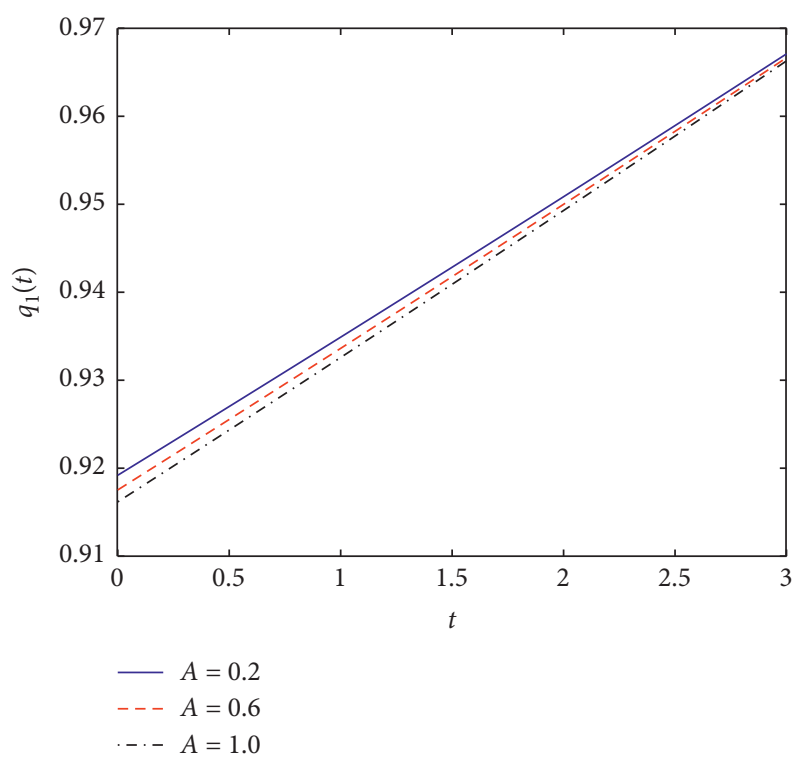

Figure 15: The effect of $A$ on $q_{1}(t)$.

Figures 8-11 demonstrate the impacts of $A, h, \beta$, and $\omega$ on the equilibrium investment policy invested in the defaultable bond $p_{2}(t)$, respectively. Since the mechanism of action is similar to $p_{1}(t)$, we omit economic analysis here.

Figures 12 and 13 show that the equilibrium strategy invested in the defaultable bond $p_{2}(t)$ increases with the credit spread $\vartheta$ and decreases with the loss rate $\zeta$ because the lower credit spread $\vartheta$ or higher loss rate $\zeta$ can induce a larger potential loss. Figure 14 presents that the greater the default intensity $k^{\mathbb{P}}$ is, the less money will be invested in default bonds. This is because the greater the default intensity $k^{\mathbb{P}}$, the higher the default risk, and the insurer will naturally reduce the money of defaultable bonds.

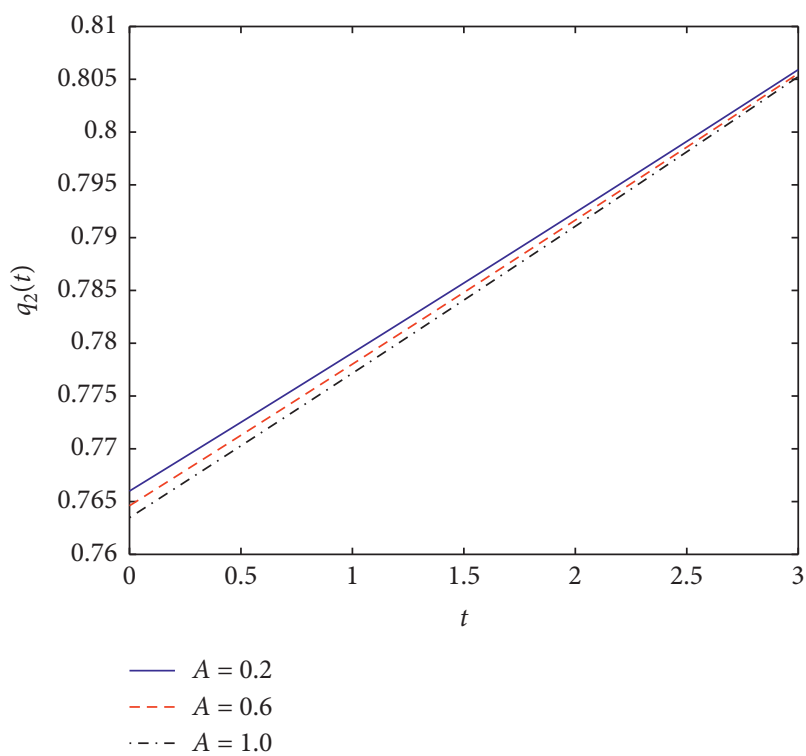

Figure 16: The effect of $A$ on $q_{2}(t)$.

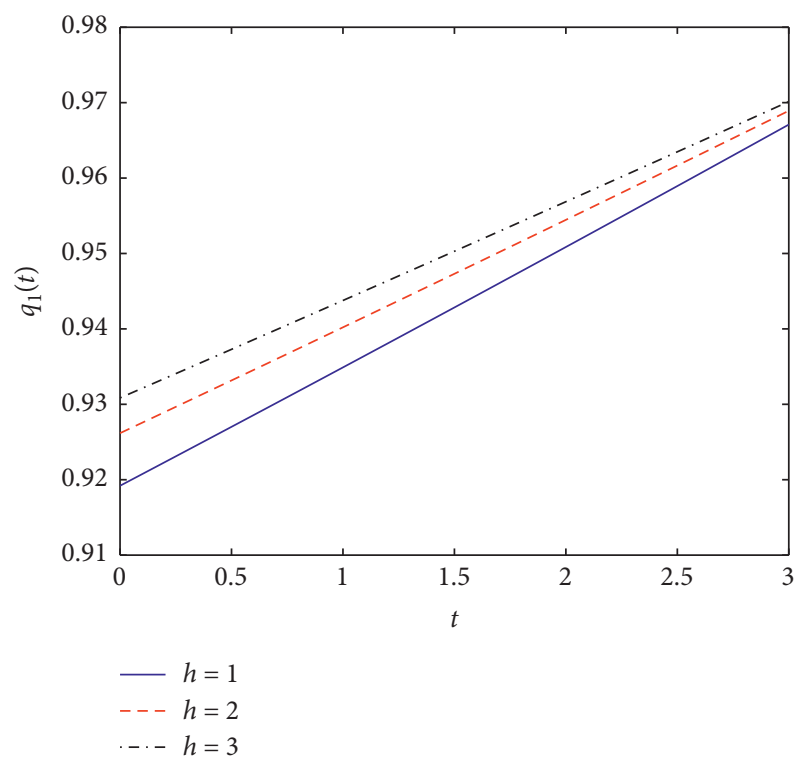

FIgure 17: The effect of $h$ on $q_{1}(t)$.

Figures 15 and 16 demonstrate the effect of parameter $A$ on reinsurance strategies $q_{1}(t)$ and $q_{2}(t)$, respectively. We can find that, with the increase of $A$, the reinsurance retention ratio $q_{1}(t)\left(q_{2}(t)\right)$ also decreases. Just as we explain why the equilibrium investment strategy decreases with respect to $A$, if $A$ is larger, the insurer pays more attention to the current wealth and wealth close to the current time, and then the insurer may face greater risk. In order to avoid the risk, the insurer will buy more reinsurance, thus reducing the retention ratio $q_{1}(t)\left(q_{2}(t)\right)$. From Figures 17 and 18, we can see that, with the increase of $h$, the reinsurance retention ratio $q_{1}(t)\left(q_{2}(t)\right)$ also increases, because the larger $h$ is, the longer the time interval for calculating the average delayed 


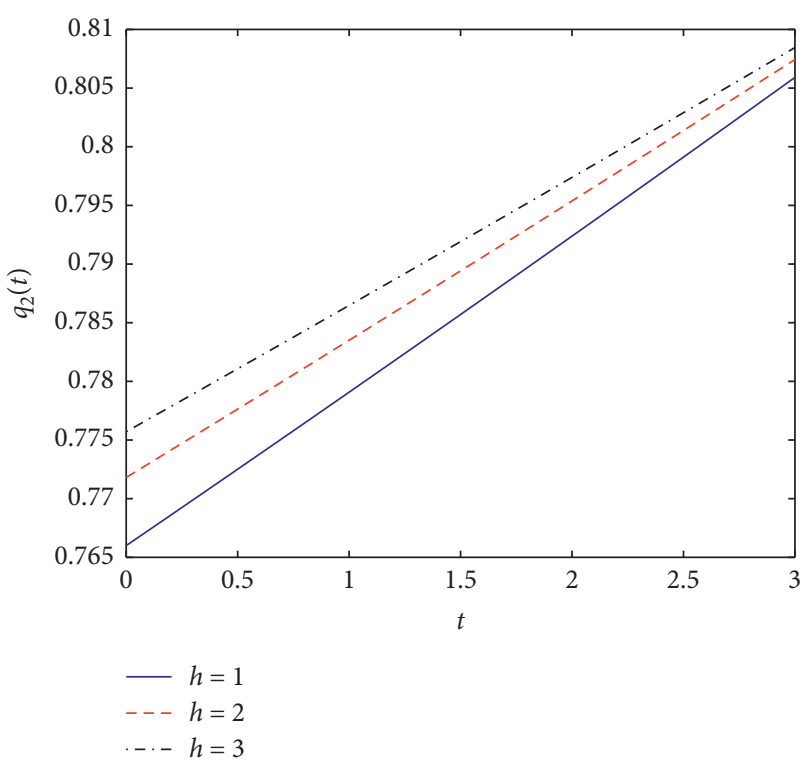

Figure 18: The effect of $h$ on $q_{2}(t)$.

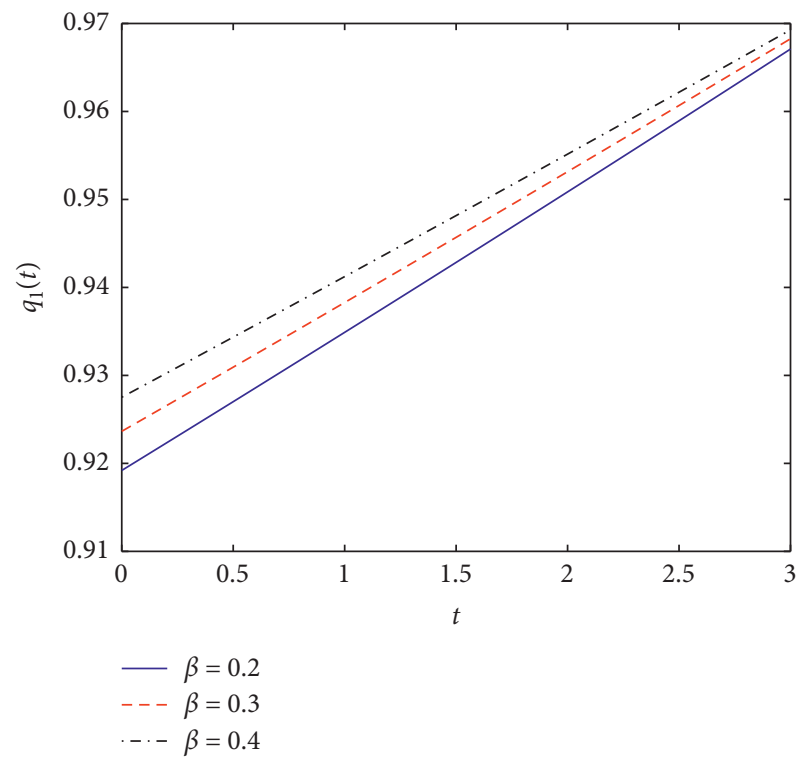

Figure 19: The effect of $\beta$ on $q_{1}(t)$.

wealth is, and the more stable the average delayed wealth is, and, therefore, the smaller the risk faced by the insurer is. In order to obtain more profits, the insurer's reinsurance retention ratio is higher. Figures 19 and 20 show the influence of parameter $\beta$ on reinsurance strategy. The results show that the level of reinsurance retention increases with respect to $\beta$. The larger $\beta$ is, the greater the average delayed wealth share weight is in the composition of terminal wealth, and the smaller the overall risk faced by the insurer is; then the insurer will reduce the purchase of reinsurance, so the retention ratio $q_{1}(t)\left(q_{2}(t)\right)$ will increase. Figures 21 and 22 reflect the impact of $\omega$ on reinsurance strategy. The results show that the reinsurance retention ratio $q_{1}(t)\left(q_{2}(t)\right)$ decreases with the increase of $\omega$, which is consistent with the economic significance of $\omega$.

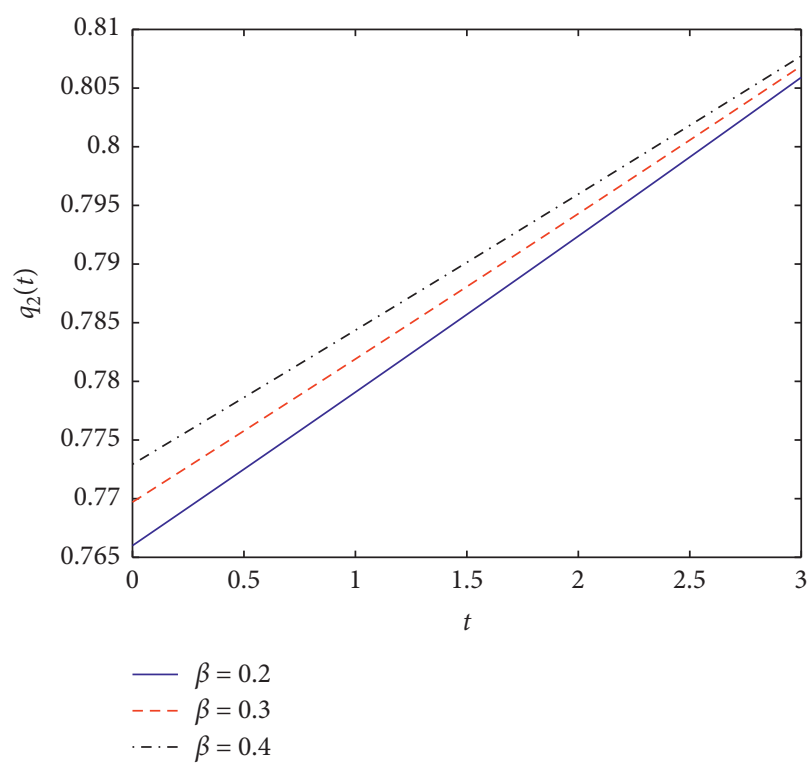

Figure 20: The effect of $\beta$ on $q_{2}(t)$.

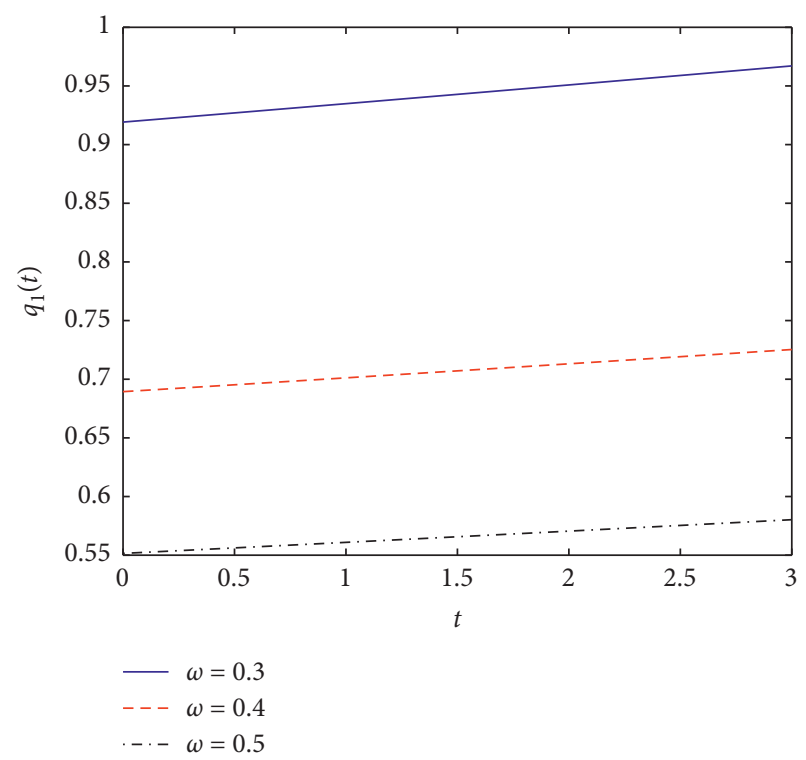

FIgURE 21: The effect of $\omega$ on $q_{1}(t)$.

Figures 23-25 depict the influence of parameters $\lambda_{1}, \lambda_{2}$, and $\lambda$ on reinsurance strategy, respectively. As can be seen from Figure 23, the larger $\lambda_{1}$, the smaller $q_{1}(t)$, and the larger $q_{2}(t)$. This is because the larger $\lambda_{1}$ is, the greater the average number of claims of the first type of insurance business is, and then the insurer will purchase more reinsurance for the first type of business, so the average amount of capital occupied by the first type of business will be less. More profits will reduce reinsurance purchases in the second category. Figure 24 shows that the larger $\lambda_{2}$, the smaller $q_{2}(t)$, and the larger $q_{1}(t)$, which is consistent with the explanation of Figure 23, because, with the increase of $\lambda$, the average number of claims in both types of the insurance 


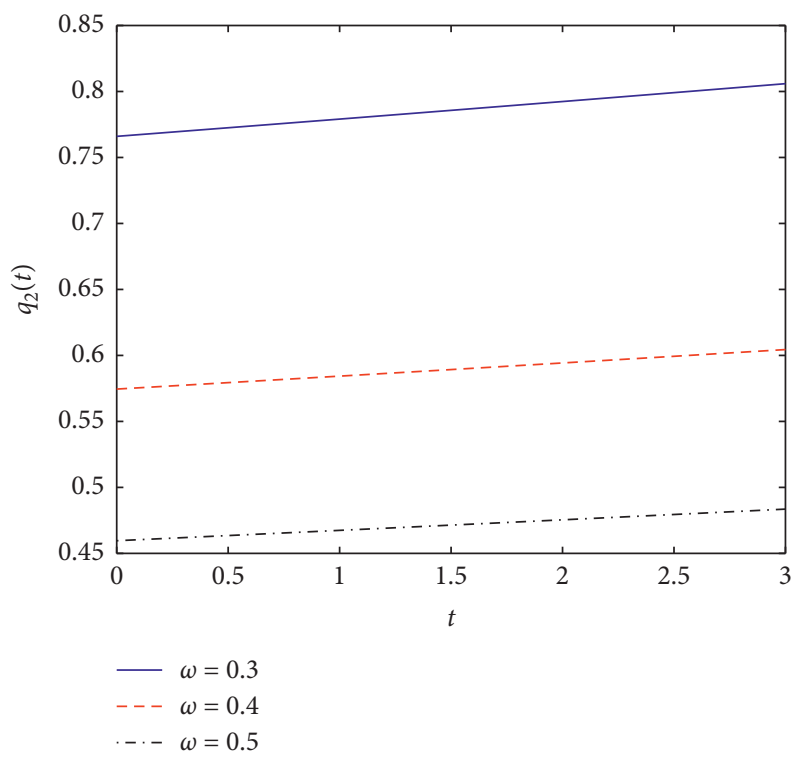

Figure 22: The effect of $\omega$ on $q_{2}(t)$.

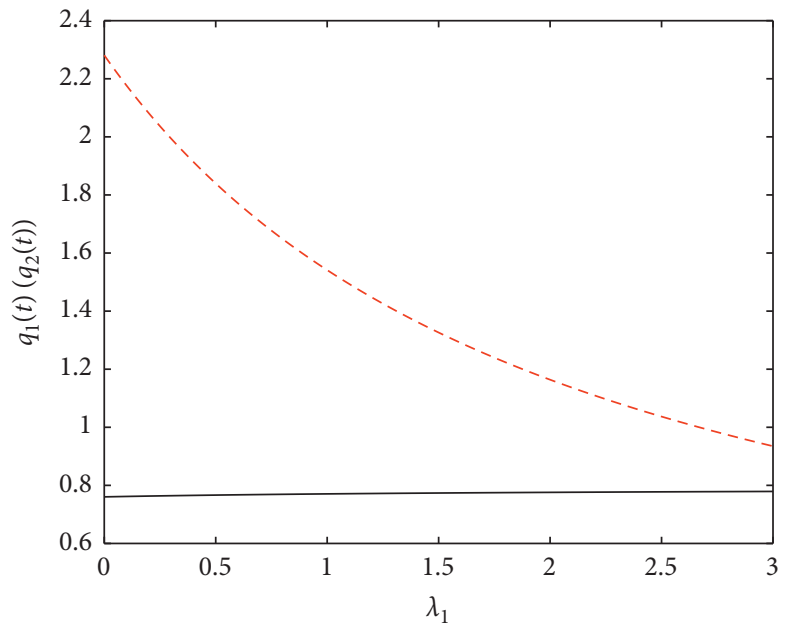

$---q_{1}$

Figure 23: The effect of $\lambda_{1}$ on $q_{1}(t)\left(q_{2}(t)\right)$.

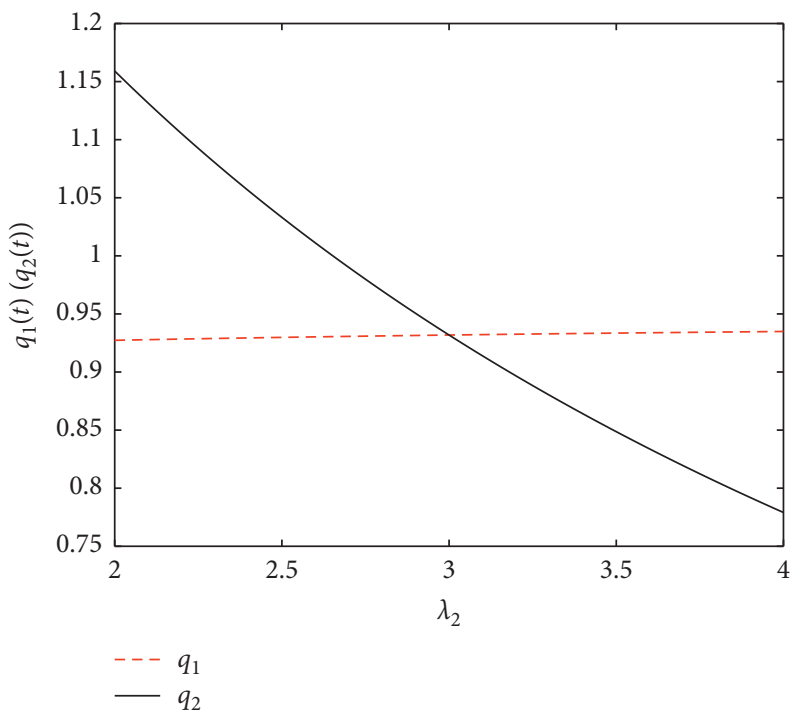

FIgURE 24: The effect of $\lambda_{2}$ on $q_{1}(t)\left(q_{2}(t)\right)$. 


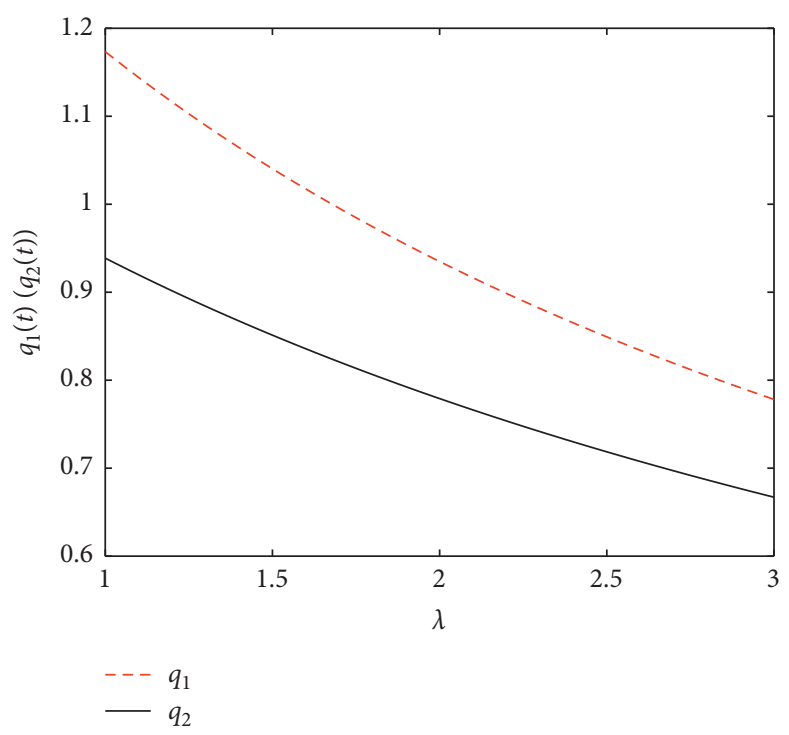

Figure 25: The effect of $\lambda$ on $q_{1}(t)\left(q_{2}(t)\right)$.

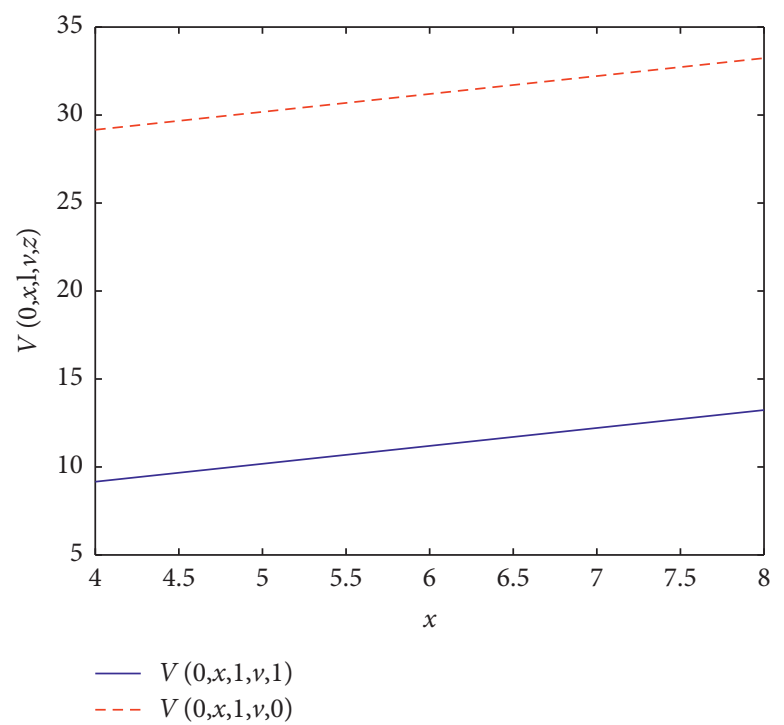

Figure 26: Equilibrium value function with respect to $x$.

business will increase, and the insurer will buy more reinsurance. Therefore, as shown in Figure 25, $q_{1}(t)\left(q_{2}(t)\right)$ decreases. From Figure 26, we can find that the postdefault equilibrium value functions $V(0, x, l, v, 1)$ are lower than the predefault equilibrium value functions $V(0, x, l, v, 1)$ because the default event induces the loss of the insurer's objective.

\section{Conclusion}

In this paper, we study the investment-reinsurance problem under Heston's SV model, which incorporates default risk, delay, and common shocks. Applying stochastic control theory in a game-theoretic framework, we obtain an explicit solution for the optimal time-consistent strategies. We also analyze the effect of some parameters on the optimal timeconsistent strategies by a numerical case. The main findings are as follows: (i) The equilibrium investment invested in risky asset and the defaultable bond and the equilibrium reinsurance strategy is affected by the delay parameters of the model, and the influence is consistent with the economic phenomenon in reality. (ii) The postdefault equilibrium value functions are lower than the predefault equilibrium value functions. (iii) The equilibrium efficient frontier with a delay has the same expression form as that of the classical mean-variance portfolio theory. In order to better simulate the market environment faced by insurers, moral hazard, inflation, regulatory mechanism, and no bankruptcy constraints are worth introducing. 


\section{Appendix}

\section{A. Proof of Theorem 1}

Proof of Theorem 1. Suppose that the functions $V(t, x, l, v, z)$ and $g(t, x, l, v, z)$ satisfy Theorem 1 , and the optimal strategy $\pi^{*}$ in (24) is reachable. In what follows, we complete the proof of the theorem in two steps: (a) and (b).

(a) We aim to prove that

$$
\begin{aligned}
g(t, x, l, v, z)= & E_{t, x, l, v, z}\left[X^{\pi^{*}}(T)+\beta L^{\pi^{*}}(T)\right], \\
& V(t, x, l, v, z)=J\left(t, x, l, v, z ; \pi^{*}\right) .
\end{aligned}
$$

According to the conditions $\mathscr{L}^{\pi^{*}} g(t, x, l, v, z)=0$ and $g(T, x, l, v, z)=x+\beta l$ in Theorem 1 and by Dynkin's formula with delay (see Lemma 2.2 in Elsanosi et al.'s work [22]), we obtain

$$
\begin{aligned}
& E_{t, x, l, v, z}\left[g\left(T, X^{\pi^{*}}(T), L^{\pi^{*}}(T), v(T), z\right)\right] \\
& \quad=g(t, x, l, v, z)+E_{t, x, l, v, z}\left[\int_{t}^{T} \mathscr{L}^{\pi^{*}} g\left(u, X^{\pi^{*}}(u), L^{\pi^{*}}(u), v(u), z\right) \mathrm{d} u\right] \\
& \quad=g(t, x, l, v, z),
\end{aligned}
$$

where the variational operator $\mathscr{L}^{\pi}$ is defined by (22). Furthermore, we derive

$$
\begin{aligned}
g(t, x, l, v, z) & =E_{t, x, l, v, z}\left[g\left(T, X^{\pi^{*}}(T), L^{\pi^{*}}(T), v(T), z\right)\right] \\
& =E_{t, x, l, v, z}\left[X^{\pi^{*}}(T)+\beta L^{\pi^{*}}(T)\right] .
\end{aligned}
$$

$$
\sup _{\pi \in \Pi}\left\{\mathscr{L}^{\pi} V(t, x, l, v, z)-\frac{\omega}{2} \mathscr{L}^{\pi} g^{2}(t, x, l, v, z)+\omega g(t, x, l, v, z) \mathscr{L}^{\pi} g(t, x, l, v, z)\right\}=0
$$

is reached at $\pi^{*}$, by the conditions $\mathscr{L}^{\pi^{*}} g(t, x, l, v, z)=0$ and $g(T, x, l, v, z)=x+\beta l$ in Theorem 1, we rewrite extended HJB equation as $\mathscr{L}^{\pi^{*}} V(t, x, l, v, z)-\frac{\omega}{2} \mathscr{L}^{\pi^{*}} g^{2}(t, x, l, v, z)=0$
By the condition $V(T, x, l, v, z)=x+\beta l$ in Theorem 1 and Dynkin's formula with delay in Lemma 2.2 of Elsanosi et al.'s work [22], we derive

$$
\begin{aligned}
E_{t, x, l, v, z}\left[X^{\pi^{*}}(T)+\beta L^{\pi^{*}}(T)\right] & =E_{t, x, l, v, z}\left[V\left(T, X^{\pi^{*}}(T), L^{\pi^{*}}(T), v(T), z\right)\right] \\
& =V(t, x, l, v, z)+\int_{t}^{T} \mathscr{L}^{\pi^{*}} V\left(u, X^{\pi^{*}}(u), L^{\pi^{*}}(u), v(u), z\right) \mathrm{d} u .
\end{aligned}
$$

Inserting (A.5) into the above equation, we derive

$$
V(t, x, l, v, z)=E_{t, x, l, v, z}\left[X^{\pi^{*}}(T)+\beta L^{\pi^{*}}(T)\right]-\frac{\omega}{2} \int_{t}^{T} \mathscr{L}^{\pi^{*}} g^{2}\left(u, X^{\pi^{*}}(u), L^{\pi^{*}}(u), v(u), z\right) \mathrm{d} u .
$$


By terminal condition and Dynkin's formula with delay (see Lemma 2.2 in Elsanosi et al.'s work [22]), we have

$$
\begin{aligned}
& E_{t, x, l, v, z}\left[\left(X^{\pi^{*}}(T)+\beta L^{\pi^{*}}(T)\right)^{2}\right]=E_{t, x, l, v, z}\left[g^{2}\left(T, X^{\pi^{*}}(T), L^{\pi^{*}}(T), v(T), z\right)\right] \\
& =g^{2}(t, x, l, v, z)+\int_{t}^{T} \mathscr{L}^{\pi^{*}} g^{2}(u, X(u), L(u), v(u), z) \mathrm{d} u \\
& =\left(E_{t, x, l, v, z}\left[X^{\pi^{*}}(T)+\beta L^{\pi^{*}}(T)\right]\right)^{2}+\int_{t}^{T} \mathscr{L}^{\pi^{*}} g^{2}(u, X(u), L(u), v(u), z) \mathrm{d} u .
\end{aligned}
$$

That is to say, we have

$$
\operatorname{Var}_{t, x, l, v, z}\left[X^{\pi^{*}}(T)+\beta L^{\pi^{*}}(T)\right]=\int_{t}^{T} \mathscr{L}^{\pi^{*}} g^{2}(u, X(u), L(u), v(u), z) \mathrm{d} u
$$

Putting equation (A.9) into equation (A.7), we arrive at

$$
\begin{aligned}
V(t, x, l, v, z) & =E_{t, x, l, v, z}\left[X^{\pi^{*}}(T)+\beta L^{\pi^{*}}(T)\right]-\frac{\omega}{2} \operatorname{Var}_{t, x, l, v, z}\left[X^{\pi^{*}}(T)+\beta L^{\pi^{*}}(T)\right] \\
& =J\left(t, x, l, v, z ; \pi^{*}\right) .
\end{aligned}
$$

(b) We aim to show that $\pi^{*}$ is an equilibrium strategy defined in Definition 2. Note that $J(t, x, l, v, z ; \pi)=$ $E_{t, x, l, v, z}\left[X^{\pi}(T)+\beta L^{\pi}(T)\right]-$ $(\omega / 2) \operatorname{Var}_{t, x, l, v, z}\left[X^{\pi}(T)+\beta L^{\pi}(T)\right]$ and by the perturbed strategy $\pi_{\varepsilon}$ in Definition 2, we can derive

$$
\begin{aligned}
& E_{t, x, l, v, z}\left[J\left(t+\varepsilon, X^{\widehat{\pi}}(t+\varepsilon), L^{\widehat{\pi}}(t+\varepsilon), v(t+\varepsilon), z\right)\right]-J\left(t, x, l, v, z ; \pi_{\varepsilon}\right) \\
& =E_{t, x, l, v, z}\left[E_{t+\varepsilon, \widehat{X^{\pi}}(t+\varepsilon), \widehat{L^{\pi}}(t+\varepsilon), v(t+\varepsilon), z}\left[\left(X^{\pi^{*}}(T)+\beta L^{\pi^{*}}(T)\right)-\frac{\omega}{2}\left(X^{\pi^{*}}(T)+\beta L^{\pi^{*}}(T)\right)^{2}\right]\right.
\end{aligned}
$$

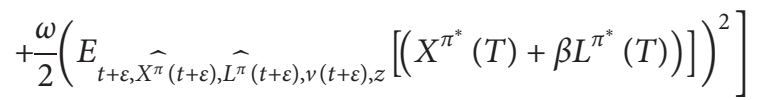

$$
\begin{aligned}
& -E_{t, x, l, v, z}\left[\left(X^{\pi^{*}}(T)+\beta L^{\pi^{*}}(T)\right)-\frac{\omega}{2}\left(X^{\pi^{*}}(T)+\beta L^{\pi^{*}}(T)\right)^{2}\right]-\frac{\omega}{2}\left(E_{t, x, l, v, z}\left[X^{\pi^{*}}(T)+\beta L^{\pi^{*}}(T)\right]\right)^{2}
\end{aligned}
$$

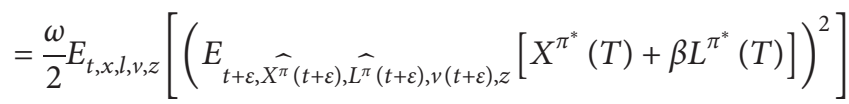

$$
\begin{aligned}
& -\frac{\omega}{2}\left(E_{t, x, l, v, z}\left[E_{t+\varepsilon, X^{\pi}(t+\varepsilon), L^{\pi}(t+\varepsilon), v(t+\varepsilon), z}\left[X^{\pi^{*}}(T)+\beta L^{\pi^{*}}(T)\right]\right]\right)^{2} \\
& =\frac{\omega}{2} E_{t, x, l, v, z}\left[g^{2}\left(t+\varepsilon, X^{\hat{\pi}}(t+\varepsilon), L^{\hat{\pi}}(t+\varepsilon), v(t+\varepsilon), z\right)\right] \\
& -\frac{\omega}{2}\left(E\left[t, x, l, v, z\left[g\left(t+\varepsilon, X^{\widehat{\pi}}(t+\varepsilon), L^{\tilde{\pi}}(t+\varepsilon), v(t+\varepsilon), z\right)\right]\right]\right)^{2} .
\end{aligned}
$$


From the above equation, we arrive at

$$
\begin{aligned}
J\left(t, x, l, v, z ; \pi_{\varepsilon}\right)= & E_{t, x, l, v, z}\left[J\left(t+\varepsilon, X^{\hat{\pi}}(t+\varepsilon), L^{\widehat{\pi}}(t+\varepsilon), v(t+\varepsilon), z ; \pi_{\varepsilon}\right)\right] \\
& -\frac{\omega}{2} E_{t, x, l, v, z}\left[g^{2}\left(t+\varepsilon, X^{\tilde{\pi}}(t+\varepsilon), L^{\tilde{\pi}}(t+\varepsilon), v(t+\varepsilon), z\right)\right] \\
& +\frac{\omega}{2}\left(E_{t, x, l, v, z}\left[g\left(t+\varepsilon, X^{\tilde{\pi}}(t+\varepsilon), L^{\hat{\pi}}(t+\varepsilon), v(t+\varepsilon), z\right)\right]\right)^{2} .
\end{aligned}
$$

By substituting extra terms $V(t, x, l, v, z)$ and $g^{2}(t, x, l, v, z)$ into the above equation and applying the result in (a), we derive

$$
\begin{aligned}
J\left(t, x, l, v, z ; \pi_{\varepsilon}\right)= & E_{t, x, l, v, z}\left[V\left(t+\varepsilon, X^{\tilde{\pi}}(t+\varepsilon), L^{\pi}(t+\varepsilon), v(t+\varepsilon), z\right)\right] \\
& -\frac{\omega}{2}\left\{E_{t, x, l, v, z}\left[g^{2}\left(t+\varepsilon, X^{\widehat{\pi}}(t+\varepsilon), L^{\widehat{\pi}}(t+\varepsilon), v(t+\varepsilon), z\right)\right]-g^{2}(t, x, l, v, z)\right\} \\
& +\frac{\omega}{2}\left\{\left(E_{t, x, l, v, z}\left[g\left(t+\varepsilon, X^{\hat{\pi}}(t+\varepsilon), L^{\tilde{\pi}}(t+\varepsilon), v(t+\varepsilon), z\right)\right]\right)^{2}-g^{2}(t, x, l, v, z)\right\} .
\end{aligned}
$$

For any $\pi \in \Pi$, small enough $\varepsilon>0$, and $\Phi \in \mathscr{C}^{1,2,1,2}([0, T] \times \mathbb{R} \times \mathbb{R} \times \mathbb{R} \times 0,1)$, we define the following operator:

$$
\mathscr{L}_{\varepsilon}^{\pi} \Phi(t, x, l, v, z)=E_{t, x, l, v, z}\left[\Phi\left(t+\varepsilon, X^{\pi}(t+\varepsilon), L^{\pi}(t+\varepsilon), v(t+\varepsilon), z\right)\right]-\Phi(t, x, l, v, z),
$$

For $\varepsilon>0$, we have

$$
\lim _{\mathcal{\varepsilon} \downarrow 0} \frac{\mathscr{L}_{\varepsilon}^{\pi} \Phi(t, x, l, v, z)}{\varepsilon}=\mathscr{L}^{\pi} \Phi(t, x, l, v, z) .
$$

$$
\begin{aligned}
J\left(t, x, l, v, z ; \pi_{\varepsilon}\right)= & V(t, x, l, v, z)+\mathscr{L}_{\varepsilon}^{\hat{\pi}} V(t, x, l, v, z)-\frac{\omega}{2} \mathscr{L}_{\varepsilon}^{\hat{\pi}} g^{2}(t, x, l, v, z) \\
& +\frac{\omega}{2}\left\{\left(E_{t, x, l, v, z}\left[g\left(t+\varepsilon, X^{\pi}(t+\varepsilon), L^{\pi}(t+\varepsilon), v(t+\varepsilon), z\right)\right]\right)^{2}-g^{2}(t, x, l, v, z)\right\} .
\end{aligned}
$$

By Dynkin's formula with delay (see Lemma 2.2 in Elsanosi et al.'s work [22]), we derive

$$
\begin{aligned}
& E_{t, x, l, v, z}\left[g\left(t+\varepsilon, X^{\hat{\pi}}(t+\varepsilon), L^{\hat{\pi}}(t+\varepsilon), v(t+\varepsilon), z\right)\right] \\
& \quad=g(t, x, l, v, z)+E_{t, x, l, v, z}\left[\int_{t}^{t+\varepsilon} \mathscr{L}^{\hat{\pi}} g\left(u, X^{\hat{\pi}}(u), L^{\widehat{\pi}}(u), v(u), z\right) \mathrm{d} u\right],
\end{aligned}
$$


which implies

$$
\begin{gathered}
\left\{E_{t, x, l, v, z}\left[g\left(t+\varepsilon, X^{\widehat{\pi}}(t+\varepsilon), L^{\widehat{\pi}}(t+\varepsilon), v(t+\varepsilon), z\right)\right]\right\}^{2}-g^{2}(t, x, l, v, z) \\
=2 g(t, x, l, v, z) E_{t, x, l, v, z}\left[\int_{t}^{t+\varepsilon} \mathscr{L}^{\widehat{\pi}} g\left(u, X^{\hat{\pi}}(u), L^{\widehat{\pi}}(u), v(u), z\right) \mathrm{d} u\right] \\
+\left\{E_{t, x, l, v, z}\left[\int_{t}^{t+\varepsilon} \mathscr{L}^{\tilde{\pi}} g\left(u, X^{\hat{\pi}}(u), L^{\hat{\pi}}(u), v(u), z\right) \mathrm{d} u\right]\right\}^{2} .
\end{gathered}
$$

Substituting equation (A.18) into equation (A.16), we have

$$
\begin{array}{r}
J\left(t, x, l, v, z ; \pi_{\varepsilon}\right)=V(t, x, \\
+\omega g(t, x) \\
+\frac{\omega}{2}\{t \\
\text { the extended HJB equation in }(23), \\
\widehat{\mathscr{\pi}} V(t, x, l, v, z)-\frac{\omega}{2} \widehat{\mathscr{L}}^{\hat{\pi}} g^{2}(t, x, l, v, z) \\
+\omega g(t, x, l, v, z) \mathscr{L}^{\hat{\pi}} g(t, x, l, v, z) \leq 0 .
\end{array}
$$

Together with equation (A.15), we have at

$$
\mathscr{L}_{\varepsilon}^{\hat{\pi}} V(t, x, l, v, z)-\frac{\omega}{2} \mathscr{L}_{\varepsilon}^{\tilde{\pi}} g^{2}(t, x, l, v, z)+\omega g(t, x, l, v, z)
$$

(23), we can obtain

$$
\times E_{t, x, l, v, z}\left[\int_{t}^{t+\varepsilon} \mathscr{L}^{\widehat{\pi}} g\left(u, X^{\widehat{\pi}}(u), L^{\widehat{\pi}}(u), v(u), z\right) \mathrm{d} u\right] \leq o(\varepsilon) .
$$

Putting equation (A.21) into equation (A.19), we arrive

$$
\begin{aligned}
J\left(t, x, l, v, z ; \pi_{\varepsilon}\right) & \leq V(t, x, l, v, z)+\frac{\omega}{2}\left\{E_{t, x, l, v, z}\left[\int_{t}^{t+\varepsilon} \mathscr{L}^{\widehat{\pi}} g\left(u, X^{\widehat{\pi}}(u), L^{\widehat{\pi}}(u), v(u), z\right) \mathrm{d} u\right]\right\}^{2}+o(\varepsilon) \\
& =J\left(t, x, l, v, z ; \pi^{*}\right)+\frac{\omega}{2}\left\{E_{t, x, l, v, z}\left[\int_{t}^{t+\varepsilon} \mathscr{L}^{\widehat{\pi}} g\left(u, X^{\widehat{\pi}}(u), L^{\widehat{\pi}}(u), v(u), z\right) \mathrm{d} u\right]\right\}^{2}+o(\varepsilon) .
\end{aligned}
$$


From the above equation, it is easy to obtain

$$
\liminf _{\varepsilon \downarrow 0} \frac{J\left(t, x, l, v, z ; \pi^{*}\right)-J\left(t, x, l, v, z ; \pi_{\varepsilon}\right)}{\varepsilon} \geq 0,
$$

that is, $\pi^{*}$ is an equilibrium strategy in Definition 2.

\section{B. Proof of Theorem 2}

Proof of Theorem 2. For the postdefault $(z=1)$, by the terminal conditions of $V$ and $g$, we conjecture that $V$ and $g$ are of the following forms:

$$
\begin{aligned}
& V(t, x, l, v, 1)=H(t)(x+\beta l)+\frac{F(t)}{\omega} v+\frac{G(t)}{\omega}, \\
& g(t, x, l, v, 1)=P(t)(x+\beta l)+\frac{Q(t)}{\omega} v+\frac{R(t)}{\omega},
\end{aligned}
$$

where $\quad H(T)=P(T)=1 \quad$ and $\quad F(T)=G(T)=Q(T)=$ $R(T)=0$.

Differentiating $V$ and $g$ with respect to $t, x, l$, and $v$, we obtain

$$
\begin{aligned}
V_{t} & =H \prime(t)(x+\beta l)+\frac{F^{\prime}(t)}{\omega} v+\frac{G^{\prime}(t)}{\omega}, \\
V_{x} & =H(t), \\
V_{l} & =\beta H(t)=\beta V_{x}, \\
V_{v} & =\frac{F(t)}{\omega}, \\
V_{v v} & =V_{x x}=V_{x v}=0, \\
g_{t} & =P \prime(t)(x+\beta l)+\frac{Q^{\prime}(t)}{\omega} v+\frac{R^{\prime}(t)}{\omega}, \\
g_{x} & =P(t), \\
g_{l} & =\beta P(t)=\beta g_{x}, \\
g_{v} & =\frac{Q(t)}{\omega}, \\
g_{v v} & =g_{x x}=g_{x v}=0 .
\end{aligned}
$$

After simple calculations, we can also get

$$
\begin{aligned}
& \left\{\begin{array}{l}
E\left[V\left(t, x-q_{1} Y_{1 i}, l, v, 1\right)-V(t, x, l, v, 1)\right]=-\mu_{11} q_{1} H(t), \\
E\left[V\left(t, x-q_{2} Y_{2 i}, l, v, 1\right)-V(t, x, l, v, 1)\right]=-\mu_{21} q_{2} H(t), \\
E\left[V\left(t, x-q_{1} Y_{1 i}-q_{2} Y_{2 i}, l, v, 1\right)-V(t, x, l, v, 1)\right]=-\mu_{11} q_{1} H(t)-\mu_{21} q_{2} H(t) .
\end{array}\right. \\
& \left\{\begin{array}{l}
E\left[g^{2}\left(t, x-q_{1} Y_{1 i}, l, v, 1\right)-g^{2}(t, x, l, v, 1)\right]=\mu_{12} q_{1}^{2} P^{2}(t) \\
-2 \mu_{11} q_{1} P(t)\left[P(t)(x+\beta l)+\frac{Q(t)}{\omega} v+\frac{R(t)}{\omega}\right], \\
E\left[g^{2}\left(t, x-q_{2} Y_{2 i}, l, v, 1\right)-g^{2}(t, x, l, v, 1)\right]=\mu_{22} q_{2}^{2} P^{2}(t) \\
-2 \mu_{21} q_{2} P(t)\left[P(t)(x+\beta l)+\frac{Q(t)}{\omega} v+\frac{R(t)}{\omega}\right], \\
E\left[g^{2}\left(t, x-q_{1}(t) Y_{1 i}-q_{2}(t) Y_{2 i}, l, v, 1\right)-g^{2}(t, x, l, v, 1)\right]=\left(\mu_{12} q_{1}^{2}+\mu_{22} q_{2}^{2}\right) P^{2}(t) \\
+2 \mu_{11} \mu_{21} q_{1} q_{2} P^{2}(t)-2\left(\mu_{11} q_{1}+\mu_{21} q_{2}\right) P(t)\left[P(t)(x+\beta l)+\frac{Q(t)}{\omega} v+\frac{R(t)}{\omega}\right],
\end{array}\right. \\
& \left\{\begin{array}{l}
E\left[g\left(t, x-q_{1} Y_{1 i}, l, v, 1\right)-g(t, x, l, v, 1)\right]=-\mu_{11} q_{1} P(t), \\
E\left[g\left(t, x-q_{2} Y_{2 i}, l, v, 1\right)-g(t, x, l, v, 1)\right]=-\mu_{21} q_{2} P(t), \\
E\left[g\left(t, x-q_{1} Y_{1 i}-q_{2} Y_{2 i}, l, v, 1\right)-g(t, x, l, v, 1)\right]=-\mu_{11} q_{1} P(t)-\mu_{21} q_{2} P(t) .
\end{array}\right.
\end{aligned}
$$


Substituting the above results into (25), we derive

$$
\left\{\begin{array}{l}
\sup _{\pi \in \Pi}\left\{H^{\prime}(t)(x+\beta l)+\frac{F^{\prime}(t)}{\omega} v+\frac{G^{\prime}(t)}{\omega}+\Gamma\left(p_{1}, q_{1}, q_{2}\right) H(t)+\kappa(\alpha-v) \frac{F(t)}{\omega}-\frac{\omega}{2} p_{1}^{2} v P^{2}(t)\right. \\
\left.-\frac{\sigma^{2} v}{2 \omega} Q^{2}(t)-p_{1} \sigma v \rho Q(t) P(t)-\frac{\omega}{2}\left(b_{1}^{2} q_{1}^{2}+b_{2}^{2} q_{2}^{2}\right) P^{2}(t)-\omega \lambda \mu_{11} \mu_{21} q_{1} q_{2} P^{2}(t)\right\}=0, \\
P^{\prime}(t)(x+\beta l)+\frac{Q^{\prime}(t)}{\omega} v+\frac{R \prime(t)}{\omega}+\Gamma\left(p_{1}^{*}, q_{1}^{*}, q_{2}^{*}\right) P(t)+\kappa(\alpha-v) \frac{Q(t)}{\omega}=0, \\
H(T)=P(T)=1, \\
F(T)=G(T)=Q(T)=R(T)=0,
\end{array}\right.
$$

where

$$
\begin{aligned}
& \Gamma\left(p_{1}, q_{1}, q_{2}\right)=\left(r-\gamma_{1}-\gamma_{2}+\beta\right) x+\left(\bar{\gamma}_{1}-A \beta\right) l \\
&+\left(\gamma_{2}-\beta e^{-A h}\right) m+\delta p v+a_{1} \eta_{1} q_{1} \\
&+a_{2} \eta_{2} q_{2}+\xi_{1} a_{1}+\xi_{2} a_{2} . \\
& \text { Since } \gamma_{2}=\beta e^{-A h}, \text { we have } \\
& \Gamma\left(p_{1}, q_{1}, q_{2}\right)=\left(r-\gamma_{1}-\gamma_{2}+\beta\right) x+\left(\bar{\gamma}_{1}-A \beta\right) l \\
&+\delta p v+a_{1} \eta_{1} q_{1} \\
&+a_{2} \eta_{2} q_{2}+\xi_{1} a_{1}+\xi_{2} a_{2} .
\end{aligned}
$$

Let

$$
\begin{aligned}
\tilde{h}\left(p_{1}, q_{1}, q_{2}\right)= & \Gamma\left(p_{1}, q_{1}, q_{2}\right) H(t)+\kappa(\alpha-v) \frac{F(t)}{\omega} \\
& -\frac{\omega}{2} p_{1}^{2} v P^{2}(t)-\frac{\sigma^{2} v}{2 \omega} Q^{2}(t) \\
& -p_{1} \sigma v \rho Q(t) P(t)-\frac{\omega}{2}\left(b_{1}^{2} q_{1}^{2}+b_{2}^{2} q_{2}^{2}\right) P^{2}(t) \\
& -\omega \lambda \mu_{11} \mu_{21} q_{1} q_{2} P^{2}(t)
\end{aligned}
$$

$$
\left\{\begin{array}{l}
\frac{\partial \widetilde{h}}{\partial p_{1}}=\delta v H(t)-\omega p_{1} v P^{2}(t)-\sigma v \rho P(t) Q(t), \\
\frac{\partial \widetilde{h}}{\partial q_{1}}=a_{1} \eta_{1} H(t)-\omega P^{2}(t) b_{1}^{2} q_{1}-\omega \lambda \mu_{11} \mu_{21} q_{2} P^{2}(t), \\
\frac{\partial \widetilde{h}}{\partial q_{2}}=a_{2} \eta_{2} H(t)-\omega P^{2}(t) b_{2}^{2} q_{2}-\omega \lambda \mu_{11} \mu_{21} q_{1} P^{2}(t), \\
\frac{\partial^{2} \widetilde{h}}{\partial p_{1}^{2}}=-\omega v P^{2}(t), \\
\frac{\partial^{2} \widetilde{h}}{\partial p_{1} \partial q_{1}}=\frac{\partial^{2} \widetilde{h}}{\partial p \partial q_{2}}=0 \\
\frac{\partial^{2} \widetilde{h}}{\partial q_{1}^{2}}=-\omega P^{2}(t) b_{1}^{2} \\
\frac{\partial^{2} \widetilde{h}}{\partial q_{2}^{2}}=-\omega P^{2}(t) b_{2}^{2} \\
\frac{\partial q_{2}}{2}
\end{array}\right.
$$

Differentiating the function $\widetilde{h}\left(p_{1}, q_{1}, q_{2}\right)$ with respect to $p_{1}, q_{1}$, and $q_{2}$, respectively, we arrive at

From (B.9), we obtain the following Hessian matrix: 


$$
\text { Hessian }=\left(\begin{array}{ccc}
\frac{\partial^{2} \widetilde{h}}{\partial p_{1}^{2}} & \frac{\partial^{2} \widetilde{h}}{\partial p_{1} \partial q_{1}} & \frac{\partial^{2} \widetilde{h}}{\partial p_{1} \partial q_{2}} \\
\frac{\partial^{2} \widetilde{h}}{\partial p_{1} \partial q_{1}} & \frac{\partial^{2} \widetilde{h}}{\partial q_{1}^{2}} & \frac{\partial^{2} \widetilde{h}}{\partial q_{1} \partial q_{2}} \\
\frac{\partial^{2} \widetilde{h}}{\partial p_{1} \partial q_{2}} & \frac{\partial^{2} \widetilde{h}}{\partial q_{1} \partial q_{2}} & \frac{\partial^{2} \widetilde{h}}{\partial q_{2}^{2}}
\end{array}\right)=-\omega P^{2}(t) \mathbf{B},
$$

where

$$
\mathbf{B}=\left(\begin{array}{ccc}
v & 0 & 0 \\
0 & b_{1}^{2} & \lambda \mu_{11} \mu_{21} \\
0 & \lambda \mu_{11} \mu_{21} & b_{2}^{2}
\end{array}\right) .
$$

It is easy to prove that matrix $\mathbf{B}$ is positive definite and noting that $P(t)>0$ (see (B.13)), the Hessian matrix is negative definite. Therefore, the point $\left(\widehat{p}_{1}, \widehat{q}_{1}, \widehat{q}_{2}\right)$ with the maximum value of function $\widetilde{h}\left(p_{1}, q_{1}, q_{2}\right)$ satisfies the following equations:

$$
\left\{\begin{array}{l}
\delta v H(t)-\omega p_{1} v P^{2}(t)-\sigma v \rho P(t) Q(t)=0, \\
a_{1} \eta_{1} H(t)-\omega P^{2}(t) b_{1}^{2} q_{1}-\omega \lambda \mu_{11} \mu_{21} q_{2} P^{2}(t)=0, \\
a_{2} \eta_{2} H(t)-\omega P^{2}(t) b_{2}^{2} q_{2}-\omega \lambda \mu_{11} \mu_{21} q_{1} P^{2}(t)=0 .
\end{array}\right.
$$

Solving the above equations, we arrive at

$$
\left\{\begin{array}{l}
\widehat{p}_{1}(t)=\frac{\delta H(t)-\sigma \rho Q(t) P(t)}{\omega P^{2}(t)}, \\
\hat{q}_{1}(t)=n_{1} \frac{H(t)}{\omega P^{2}(t)} \\
\widehat{q}_{2}(t)=n_{2} \frac{H(t)}{\omega P^{2}(t)}
\end{array}\right.
$$

where

$$
\begin{aligned}
& n_{1}=\frac{a_{1} \eta_{1} b_{2}^{2}-a_{2} \eta_{2} \lambda \mu_{11} \mu_{21}}{b_{1}^{2} b_{2}^{2}-\lambda^{2} \mu_{11}^{2} \mu_{21}^{2}}, \\
& n_{2}=\frac{a_{2} \eta_{2} b_{1}^{2}-a_{1} \eta_{1} \lambda \mu_{11} \mu_{21}}{b_{1}^{2} b_{2}^{2}-\lambda^{2} \mu_{11}^{2} \mu_{21}^{2}} .
\end{aligned}
$$

Substituting $\left(\widehat{p}_{1}, \widehat{q}_{1}, \widehat{q}_{2}\right)$ back into (B.5), we derive

$$
\begin{aligned}
& H_{\prime}(t)(x+\beta l)+\frac{F_{\prime}(t)}{\omega} v+\frac{G_{\prime}^{\prime}(t)}{\omega}+\left[\left(r-\gamma_{1}-\gamma_{2}+\beta\right) x+\left(\bar{\gamma}_{1}-A \beta\right) l+\xi_{1} a_{1}+\xi_{2} a_{2}\right] H(t) \\
& +\left[\frac{1}{2} \delta^{2} v+a_{1} \eta_{1} n_{1}+a_{2} \eta_{2} n_{2}-\frac{1}{2}\left(b_{1}^{2} n_{1}^{2}+b_{2}^{2} n_{2}^{2}\right)-\lambda \mu_{11} \mu_{21} n_{1} n_{2}\right] \frac{H^{2}(t)}{\omega P^{2}(t)} \\
& +\kappa(\alpha-v) \frac{F(t)}{\omega}-\frac{\sigma^{2} v}{2 \omega} Q^{2}(t)\left(1-\rho^{2}\right)-\frac{\delta \sigma \rho v H(t) Q(t)}{\omega P(t)}=0, \\
& \quad P_{\prime}(t)(x+\beta l)+\frac{Q^{\prime}(t)}{\omega} v+\frac{R \prime(t)}{\omega}+\left[\left(r-\gamma_{1}-\gamma_{2}+\beta\right) x+\left(\bar{\gamma}_{1}-A \beta\right) l+\xi_{1} a_{1}+\xi_{2} a_{2}\right] P(t) \\
& +\left(\delta^{2} v+a_{1} \eta_{1} n_{1}+a_{2} \eta_{2} n_{2}\right) \frac{H(t)}{\omega P(t)}+\kappa(\alpha-v) \frac{Q(t)}{\omega}-\delta v \sigma \rho \frac{Q(t)}{\omega}=0 .
\end{aligned}
$$

According to $\bar{\gamma}_{1}-A \beta=\left(r-\gamma_{1}-\gamma_{2}+\beta\right) \beta$, it is easy to obtain that $\left(r-\gamma_{1}-\gamma_{2}+\beta\right) x+\left(\bar{\gamma}_{1}-A \beta\right) l=(r-$ $\left.\gamma_{1}-\gamma_{2}+\beta\right)(x+\beta l)$. Hence, the above equations can be rewritten as 


$$
\begin{aligned}
& {\left[H^{\prime}(t)+\left(r-\gamma_{1}-\gamma_{2}+\beta\right)\right](x+\beta l)+\frac{F^{\prime}(t)}{\omega} v+\frac{G^{\prime}(t)}{\omega}+\left(\xi_{1} a_{1}+\xi_{2} a_{2}\right) H(t)+\left[\frac{1}{2} \delta^{2} v+a_{1} \eta_{1} n_{1}\right.} \\
& \left.+a_{2} \eta_{2} n_{2}-\frac{1}{2}\left(b_{1}^{2} n_{1}^{2}+b_{2}^{2} n_{2}^{2}\right)-\lambda \mu_{11} \mu_{21} n_{1} n_{2}\right] \frac{H^{2}(t)}{\omega P^{2}(t)}+\kappa(\alpha-v) \frac{F(t)}{\omega}-\frac{\sigma^{2} v}{2 \omega} Q^{2}(t)\left(1-\rho^{2}\right) \\
& \quad-\frac{\delta \sigma \rho v H(t) Q(t)}{\omega P(t)}=0 \\
& \quad \cdot\left[P \prime(t)+\left(r-\gamma_{1}-\gamma_{2}+\beta\right)\right](x+\beta l)+\frac{Q^{\prime}(t)}{\omega} v+\frac{R \prime(t)}{\omega}+\left(\xi_{1} a_{1}+\xi_{2} a_{2}\right) P(t)+\left(\delta^{2} v+a_{1} \eta_{1} n_{1}\right. \\
& \left.+a_{2} \eta_{2} n_{2}\right) \frac{H(t)}{\omega P(t)}+\kappa(\alpha-v) \frac{Q(t)}{\omega}-\delta v \sigma \rho \frac{Q(t)}{\omega}=0 .
\end{aligned}
$$

By separating variables, we can obtain the following differential equations:

$$
\begin{aligned}
& H^{\prime}(t)+\left(r-\gamma_{1}-\gamma_{2}+\beta\right) H(t)=0, \quad H(T)=1, \\
& P \prime(t)+\left(r-\gamma_{1}-\gamma_{2}+\beta\right) P(t)=0, \quad P(T)=1, \\
& Q^{\prime}(t)-(\kappa+\delta \sigma \rho) Q(t)+\delta^{2}=0, \quad Q(T)=0, \\
& F^{\prime}(t)-\kappa F(t)-\frac{\sigma^{2}}{2} Q^{2}(t)\left(1-\rho^{2}\right)-\delta \sigma \rho Q(t)+\frac{\delta^{2}}{2}=0, \quad F(T)=0, \\
& R^{\prime}(t)+\omega\left(\xi_{1} a_{1}+\xi_{2} a_{2}\right) P(t)+\kappa \alpha Q(t)+\left(a_{1} \eta_{1} n_{1}+a_{2} \eta_{2} n_{2}\right)=0, \quad R(T)=0, \\
& G^{\prime}(t)+\omega\left(\xi_{1} a_{1}+\xi_{2} a_{2}\right) H(t)+\kappa \alpha F(t)+\left(a_{1} \eta_{1} n_{1}+a_{2} \eta_{2} n_{2}-\frac{1}{2}\left(b_{1}^{2} n_{1}^{2}+b_{2}^{2} n_{2}^{2}\right)-\lambda \mu_{11} \mu_{21} n_{1} n_{2}\right)=0, \quad G(T)=0 \\
& H(t)=P(t)=e^{\left(r-\gamma_{1}-\gamma_{2}+\beta\right)(T-t)}, \\
& G(t)=\frac{\omega\left(\xi_{1} a_{1}+\xi_{2} a_{2}\right)}{r-\gamma_{1}-\gamma_{2}+\beta}\left[e^{\left(r-\gamma_{1}-\gamma_{2}+\beta\right)(T-t)}-1\right] \\
& +\left(a_{1} \eta_{1} n_{1}+a_{2} \eta_{2} n_{2}\right. \\
& \left.-\frac{1}{2}\left(b_{1}^{2} n_{1}^{2}+b_{2}^{2} n_{2}^{2}\right)-\lambda \mu_{11} \mu_{21} n_{1} n_{2}\right)(T-t) \\
& +\kappa \alpha \int_{t}^{T} F(s) \mathrm{d} s
\end{aligned}
$$

$$
Q(t)=\left\{\begin{array}{l}
\frac{\delta^{2}}{\kappa+\delta \sigma \rho}\left[1-e^{(\kappa+\delta \sigma \rho)(t-T)}\right], \quad \kappa+\delta \sigma \rho \neq 0, \\
\delta^{2}(T-t), \quad \kappa+\delta \sigma \rho=0
\end{array}\right.
$$

$$
F(t)=e^{\kappa t} \int_{t}^{T} e^{-\kappa u}\left[-\frac{\sigma^{2}}{2} Q^{2}(u)\left(1-\rho^{2}\right)-\delta \sigma \rho Q(u)+\frac{\delta^{2}}{2}\right] \mathrm{d} u,
$$

$$
\begin{aligned}
R(t)= & \frac{\omega\left(\xi_{1} a_{1}+\xi_{2} a_{2}\right)}{r-\gamma_{1}-\gamma_{2}+\beta}\left[e^{\left(r-\gamma_{1}-\gamma_{2}+\beta\right)(T-t)}-1\right] c r \\
& +\kappa \alpha \int_{t}^{T} Q(s) \mathrm{d} s,
\end{aligned}
$$

Similarly, for the predefault case, we conjecture that $V$ and $g$ are of the following forms:

$$
\begin{aligned}
& V(t, x, l, v, 0)=H_{0}(t)(x+\beta l)+\frac{F_{0}(t)}{\omega} v+\frac{G_{0}(t)}{\omega}, \\
& g(t, x, l, v, 0)=P_{0}(t)(x+\beta l)+\frac{Q_{0}(t)}{\omega} v+\frac{R_{0}(t)}{\omega},
\end{aligned}
$$

where $V(T, x, l, v, 0)=x+\beta l$ and $g(T, x, l, v, 0)=x+\beta l$.

Similarly, differentiating $V$ and $g$ with respect to $t, x, l$, and $v$, we derive 


$$
\begin{aligned}
V_{t} & =H_{0}^{\prime}(t)(x+\beta l)+\frac{F_{0}^{\prime}(t)}{\omega} v+\frac{G_{0}^{\prime}(t)}{\omega}, \\
V_{x} & =H_{0}(t), \\
V_{l} & =\beta H_{0}(t)=\beta V_{x}, \\
V_{v} & =\frac{F_{0}(t)}{\omega}, \\
V_{v v} & =V_{x x}=V_{x v}=0,
\end{aligned}
$$

$$
\begin{aligned}
g_{t} & =P_{0}^{\prime}(t)(x+\beta l)+\frac{Q_{0}^{\prime}(t)}{\omega} v+\frac{R_{0}^{\prime}(t)}{\omega}, \\
g_{x} & =P_{0}(t), \\
g_{l} & =\beta P_{0}(t)=\beta g_{x}, \\
g_{v} & =\frac{Q_{0}(t)}{\omega} \\
g_{v v} & =g_{x x}=g_{x v}=0,
\end{aligned}
$$

and, after simple calculations, we can also arrive at

$$
\begin{aligned}
& \left\{\begin{array}{l}
E\left[V\left(t, x-q_{1} Y_{1 i}, l, v, 0\right)-V(t, x, l, v, 0)\right]=-\mu_{11} q_{1} H_{0}(t), \\
E\left[V\left(t, x-q_{2} Y_{2 i}, l, v, 0\right)-V(t, x, l, v, 0)\right]=-\mu_{21} q_{2} H_{0}(t), \\
E\left[V\left(t, x-q_{1} Y_{1 i}-q_{2} Y_{2 i}, l, v, 0\right)-V(t, x, l, v, 0)\right]=-\mu_{11} q_{1} H_{0}(t)-\mu_{21} q_{2} H_{0}(t) .
\end{array}\right. \\
& \left\{\begin{array}{l}
E\left[g^{2}\left(t, x-q_{1} Y_{1 i}, l, v, 0\right)-g^{2}(t, x, l, v, 0)\right]=\mu_{12} q_{1}^{2} P_{0}^{2}(t) \\
-2 \mu_{11} q_{1} P_{0}(t)\left[P_{0}(t)(x+\beta l)+\frac{Q_{0}(t)}{\omega} v+\frac{R_{0}(t)}{\omega}\right], \\
E\left[g^{2}\left(t, x-q_{2} Y_{2 i}, l, v, 0\right)-g^{2}(t, x, l, v, 0)\right]=\mu_{22} q_{2}^{2} P_{0}^{2}(t) \\
-2 \mu_{21} q_{2} P_{0}(t)\left[P_{0}(t)(x+\beta l)+\frac{Q_{0}(t)}{\omega} v+\frac{R_{0}(t)}{\omega}\right], \\
E\left[g^{2}\left(t, x-q_{1}(t) Y_{1 i}-q_{2}(t) Y_{2 i}, l, v, 0\right)-g^{2}(t, x, l, v, 0)\right]=\left(\mu_{12} q_{1}^{2}+\mu_{22} q_{2}^{2}\right) P_{0}^{2}(t) \\
+2 \mu_{11} \mu_{21} q_{1} q_{2} P_{0}^{2}(t)-2\left(\mu_{11} q_{1}+\mu_{21} q_{2}\right) P_{0}(t)\left[P_{0}(t)(x+\beta l)+\frac{Q_{0}(t)}{\omega} v+\frac{R_{0}(t)}{\omega}\right],
\end{array}\right. \\
& \left\{\begin{array}{l}
E\left[g\left(t, x-q_{1} Y_{1 i}, l, v, 0\right)-g(t, x, l, v, 0)\right]=-\mu_{11} q_{1} P_{0}(t), \\
E\left[g\left(t, x-q_{2} Y_{2 i}, l, v, 0\right)-g(t, x, l, v, 0)\right]=-\mu_{21} q_{2} P_{0}(t), \\
E\left[g\left(t, x-q_{1} Y_{1 i}-q_{2} Y_{2 i}, l, v, 0\right)-g(t, x, l, v, 0)\right]=-\mu_{11} q_{1} P_{0}(t)-\mu_{21} q_{2} P_{0}(t) .
\end{array}\right. \\
& \left\{\begin{array}{l}
V\left(t, x-\zeta p_{2}, l, v, 1\right)-V(t, x, l, v, 0)=H(t)\left(x+\beta l-\zeta p_{2}\right)-H_{0}(t)(x+\beta l) \\
+\frac{v}{\omega}\left(F(t)-F_{0}(t)\right)+\frac{G(t)-G_{0}(t)}{\omega}, \\
g\left(t, x-\zeta p_{2}, l, v, 1\right)-g(t, x, l, v, 0)=P(t)\left(x+\beta l-\zeta p_{2}\right)-P_{0}(t)(x+\beta l) \\
+\frac{v}{\omega}\left(Q(t)-Q_{0}(t)\right)+\frac{R(t)-R_{0}(t)}{\omega} .
\end{array}\right.
\end{aligned}
$$


Putting the above results into (26) and noting that $\gamma_{2}=\beta e^{-A h}$, we derive

$$
\begin{aligned}
& \sup _{\pi \in \Pi}\{ H_{0}^{\prime}(t)(x+\beta l)+\frac{F_{0}^{\prime}(t)}{\omega} v+\frac{G_{0}^{\prime}(t)}{\omega}+\Gamma\left(p_{1}, p_{2}, q_{1}, q_{2}\right) H_{0}(t)+\kappa(\alpha-v) \frac{F_{0}(t)}{\omega}-\frac{\omega}{2} p_{1}^{2} v P_{0}^{2}(t) \\
&-\frac{\sigma^{2} v}{2 \omega} Q_{0}^{2}(t)-p_{1} \sigma v \rho Q_{0}(t) P_{0}(t)-\frac{\omega}{2}\left(b_{1}^{2} q_{1}^{2}+b_{2}^{2} q_{2}^{2}\right) P_{0}^{2}(t)-\omega \lambda \mu_{11} \mu_{21} q_{1} q_{2} P_{0}^{2}(t) \\
&+\left[H(t)\left(x+\beta l-\zeta p_{2}\right)-H_{0}(t)(x+\beta l)+\frac{v}{\omega}\left(F(t)-F_{0}(t)\right)+\frac{G(t)-G_{0}(t)}{\omega}\right] k^{\mathbb{P}} \\
&-\frac{\omega}{2}\left[P(t)\left(x+\beta l-\zeta p_{2}\right)-P_{0}(t)(x+\beta l)+\frac{v}{\omega}\left(Q(t)-Q_{0}(t)\right)+\frac{R(t)-R_{0}(t)}{\omega}\right]^{2} k^{\mathbb{P}}=0 \\
& P_{0}^{\prime}(t)(x+\beta l)+\frac{Q_{0}^{\prime}(t)}{\omega} v+\frac{R_{0}^{\prime}(t)}{\omega}+\Gamma\left(p_{1}^{*}, p_{2}^{*}, q_{1}^{*}, q_{2}^{*}\right) P_{0}(t)+\kappa(\alpha-v) \frac{Q_{0}(t)}{\omega} \\
&+P(t)\left(x+\beta l-\zeta p_{2}\right)-P_{0}(t)(x+\beta l)+\frac{v}{\omega}\left(Q(t)-Q_{0}(t)\right)+\frac{R(t)-R_{0}(t)}{\omega}=0 \\
& H_{0}(T)=P_{0}(T)=1, \\
& F_{0}(T)=G_{0}(T)=Q_{0}(T)=R_{0}(T)=0,
\end{aligned}
$$

where

$$
\begin{aligned}
\Gamma\left(p_{1}, p_{2}, q_{1}, q_{2}\right)= & \left(r-\gamma_{1}-\gamma_{2}+\beta\right) x+\left(\bar{\gamma}_{1}-A \beta\right)+\delta p_{1} v+a_{1} \eta_{1} q_{1} \\
& +a_{2} \eta_{2} q_{2}+\xi_{1} a_{1}+\xi_{2} a_{2}+\vartheta p_{2} .
\end{aligned}
$$

Let

$$
\begin{aligned}
\widetilde{h}_{0}\left(p_{1}, p_{2}, q_{1}, q_{2}\right)= & \Gamma\left(p_{1}, p_{2}, q_{1}, q_{2}\right) H_{0}(t)+\kappa(\alpha-v) \frac{F_{0}(t)}{\omega}-\frac{\omega}{2} p_{1}^{2} v P_{0}^{2}(t)-\frac{\sigma^{2} v}{2 \omega} Q_{0}^{2}(t) \\
& -p_{1} \sigma v \rho Q_{0}(t) P_{0}(t)-\frac{\omega}{2}\left(b_{1}^{2} q_{1}^{2}+b_{2}^{2} q_{2}^{2}\right) P_{0}^{2}(t)-\omega \lambda \mu_{11} \mu_{21} q_{1} q_{2} P_{0}^{2}(t) \\
& +\left[H(t)\left(x+\beta l-\zeta p_{2}\right)-H_{0}(t)(x+\beta l)+\frac{v}{\omega}\left(F(t)-F_{0}(t)\right)+\frac{G(t)-G_{0}(t)}{\omega}\right] k^{\mathbb{P}} \\
& -\frac{\omega}{2}\left[P(t)\left(x+\beta l-\zeta p_{2}\right)-P_{0}(t)(x+\beta l)+\frac{v}{\omega}\left(Q(t)-Q_{0}(t)\right)+\frac{R(t)-R_{0}(t)}{\omega}\right]^{2} k^{\mathbb{P}}
\end{aligned}
$$


Differentiating the function $\widetilde{h}_{0}\left(p_{1}, p_{2}, q_{1}, q_{2}\right)$ with respect to $p_{1}, p_{2}, q_{1}$, and $q_{2}$, respectively, we derive

$$
\left\{\begin{array}{l}
\frac{\partial \widetilde{h}_{0}}{\partial p_{1}}=\delta v H_{0}(t)-\omega p_{1} v P_{0}^{2}(t)-\sigma v \rho P_{0}(t) Q_{0}(t) \\
\frac{\partial \widetilde{h}_{0}}{\partial p_{2}}=\vartheta H_{0}(t)-\zeta H(t) k^{\mathbb{P}}+\omega \zeta P(t) k^{\mathbb{P}}\left[P(t)\left(x+\beta l-\zeta p_{2}\right)-P_{0}(t)(x+\beta l)\right. \\
\left.+\frac{v}{\omega}\left(Q(t)-Q_{0}(t)\right)+\frac{R(t)-R_{0}(t)}{\omega}\right] \\
\frac{\partial \widetilde{h}_{0}}{\partial q_{1}}=a_{1} \eta_{1} H_{0}(t)-\omega P_{0}^{2}(t) b_{1}^{2} q_{1}-\omega \lambda \mu_{11} \mu_{21} q_{2} P_{0}^{2}(t) \\
\frac{\partial \widetilde{h}_{0}}{\partial q_{2}}=a_{2} \eta_{2} H_{0}(t)-\omega P_{0}^{2}(t) b_{2}^{2} q_{2}-\omega \lambda \mu_{11} \mu_{21} q_{1} P_{0}^{2}(t), \\
\frac{\partial^{2} \widetilde{h}_{0}}{\partial p_{1}^{2}}=-\omega v P_{0}^{2}(t), \\
\frac{\partial^{2} \widetilde{h}_{0}}{\partial q_{1} \partial q_{2}}=-\omega \lambda \mu_{11} \mu_{21} P_{0}^{2}(t) . \\
\frac{\partial^{2} \widetilde{h}_{0}}{\partial p_{2}^{2}}=-\omega \widetilde{h}_{0}^{2} P^{2}(t) k^{\mathbb{P}} \\
\frac{\partial^{2} \widetilde{h}_{0}}{\partial q_{2}^{2}}=-\omega P_{2}^{2}(t) b_{1}^{2}, \\
\partial p_{1} \partial q_{1}
\end{array}\right.
$$


From (B.30), we obtain the following Hessian matrix: where

$$
\text { Hessian }=\left(\begin{array}{cccc}
\frac{\partial^{2} \widetilde{h}}{\partial p_{1}^{2}} & \frac{\partial^{2} \widetilde{h}}{\partial p_{1} \partial p_{2}} & \frac{\partial^{2} \widetilde{h}}{\partial p_{1} \partial q_{1}} & \frac{\partial^{2} \widetilde{h}}{\partial p_{1} \partial q_{2}} \\
\frac{\partial^{2} \widetilde{h}}{\partial p_{2} \partial p_{1}} & \frac{\partial^{2} \widetilde{h}}{\partial p_{2}^{2}} & \frac{\partial^{2} \widetilde{h}}{\partial p_{2} \partial q_{1}} & \frac{\partial^{2} \widetilde{h}}{\partial p_{2} \partial q_{2}} \\
\frac{\partial^{2} \widetilde{h}}{\partial q_{1} \partial p_{1}} & \frac{\partial^{2} \widetilde{h}}{\partial q_{1} \partial p_{2}} & \frac{\partial^{2} \widetilde{h}}{\partial q_{1}^{2}} & \frac{\partial^{2} \widetilde{h}}{\partial q_{1} \partial q_{2}} \\
\frac{\partial^{2} \widetilde{h}}{\partial q_{2} \partial p_{1}} & \frac{\partial^{2} \widetilde{h}}{\partial q_{2} \partial p_{2}} & \frac{\partial^{2} \widetilde{h}}{\partial q_{2} \partial q_{1}} & \frac{\partial^{2} \widetilde{h}}{\partial q_{2}^{2}}
\end{array}\right)
$$

$$
\mathbf{B}_{0}=\left(\begin{array}{cccc}
v & 0 & 0 & 0 \\
0 & \zeta^{2} k^{\mathbb{P}} & 0 & 0 \\
0 & 0 & b_{1}^{2} & \lambda \mu_{11} \mu_{21} \\
0 & 0 & \lambda \mu_{11} \mu_{21} & b_{2}^{2}
\end{array}\right) .
$$

It is easy to prove that matrix $\mathbf{B}_{0}$ is positive definite and noting that $P_{0}(t)=P(t)>0$ (see (B.18) and (B.37)), the Hessian matrix is negative definite. Therefore, the point $\left(\widehat{p}_{1}, \widehat{p}_{2}, \widehat{q}_{1}, \widehat{q}_{2}\right)$ with the maximum value of function $\widetilde{h}\left(p_{1}, p_{2}, q_{1}, q_{2}\right)$ meets the following equations:

$$
\left\{\begin{array}{l}
\delta v H_{0}(t)-\omega p_{1} v P_{0}^{2}(t)-\sigma v \rho P_{0}(t) Q_{0}(t)=0, \\
\vartheta H_{0}(t)-\zeta H(t) k^{\mathbb{P}}+\omega \zeta P(t) k^{\mathbb{P}}\left[P(t)\left(x+\beta l-\zeta p_{2}\right)-P_{0}(t)(x+\beta l)\right. \\
\left.+\frac{v}{\omega}\left(Q(t)-Q_{0}(t)\right)+\frac{R(t)-R_{0}(t)}{\omega}\right]=0, \\
a_{1} \eta_{1} H_{0}(t)-\omega P_{0}^{2}(t) b_{1}^{2} q_{1}-\omega \lambda \mu_{11} \mu_{21} q_{2} P_{0}^{2}(t)=0, \\
a_{2} \eta_{2} H_{0}(t)-\omega P_{0}^{2}(t) b_{2}^{2} q_{2}-\omega \lambda \mu_{11} \mu_{21} q_{1} P_{0}^{2}(t)=0,
\end{array}\right.
$$

and then we derive

$$
\left\{\begin{array}{l}
\widehat{p}_{1}(t)=\frac{\delta H_{0}(t)-\sigma \rho Q_{0}(t) P_{0}(t)}{\omega P_{0}^{2}(t)} \\
\widehat{p}_{2}(t)=\frac{\vartheta H_{0}(t)}{\omega \zeta^{2} P^{2}(t) k^{\mathbb{P}}}-\frac{H(t)}{\omega \zeta P^{2}(t)}+\frac{x+\beta l}{\zeta}-\frac{P_{0}(t)(x+\beta l)}{\zeta P(t)}+\frac{v\left(Q(t)-Q_{0}(t)\right)}{\omega \zeta P(t)}+\frac{R(t)-R_{0}(t)}{\omega \zeta P(t)}, \\
\widehat{q}_{1}(t)=n_{1} \frac{H_{0}(t)}{\omega P_{0}^{2}(t)} \\
\widehat{q}_{2}(t)=n_{2} \frac{H_{0}(t)}{\omega P_{0}^{2}(t)} .
\end{array}\right.
$$


Noting that $\left(r-\gamma_{1}-\gamma_{2}+\beta\right) x+\left(\bar{\gamma}_{1}-A \beta\right) l=\left(r-\gamma_{1}-\right.$ $\left.\gamma_{2}+\beta\right)(x+\beta l)$ and putting the above results back into (B.12), we have

$$
\begin{aligned}
& H_{0}^{\prime}(t)(x+\beta l)+\frac{F_{0}^{\prime}(t)}{\omega} v+\frac{G_{0}^{\prime}(t)}{\omega}+\left[\left(r-\gamma_{1}-\gamma_{2}+\beta\right)(x+\beta l)+\xi_{1} a_{1}+\xi_{2} a_{2}\right] H_{0}(t) \\
& +\left[\frac{1}{2} \delta^{2} v+a_{1} \eta_{1} n_{1}+a_{2} \eta_{2} n_{2}-\frac{1}{2}\left(b_{1}^{2} n_{1}^{2}+b_{2}^{2} n_{2}^{2}\right)-\lambda \mu_{11} \mu_{21} n_{1} n_{2}\right] \frac{H_{0}^{2}(t)}{\omega P_{0}^{2}(t)}+\kappa(\alpha-v) \frac{F_{0}(t)}{\omega} \\
& -\frac{\sigma^{2} v}{2 \omega} Q_{0}^{2}(t)\left(1-\rho^{2}\right)-\frac{\delta \sigma \rho v H_{0}(t) Q_{0}(t)}{\omega P_{0}(t)}+\left(\frac{\vartheta H_{0}(t)}{\zeta}-H_{0}(t) k^{\mathbb{P}}+\frac{H(t) P_{0}(t) k^{\mathbb{P}}}{P(t)}\right. \\
& \left.-\frac{\vartheta H_{0}(t) P_{0}(t)}{P(t) \zeta}\right)(x+\beta l)+v\left(\frac{\left(F(t)-F_{0}(t)\right) k^{\mathbb{P}}}{\omega}+\frac{H(t)\left(Q_{0}(t)-Q(t)\right) k^{\mathbb{P}}}{P(t) \omega}\right. \\
& \left.+\frac{\vartheta H_{0}(t)\left(Q(t)-Q_{0}(t)\right)}{P(t) \zeta \omega}\right)+\frac{\left(G(t)-G_{0}(t) k^{\mathbb{P}}\right)}{\omega}+\frac{H(t)\left(R_{0}(t)-R(t)\right) k^{\mathbb{P}}}{P(t) \omega} \\
& +\frac{\vartheta H_{0}(t)\left(R(t)-R_{0}(t)\right)}{P(t) \zeta \omega}+\frac{H^{2}(t) k^{\mathbb{P}}}{2 P^{2}(t) \omega}-\frac{\vartheta H(t) H_{0}(t)}{P^{2}(t) \zeta \omega}+\frac{\vartheta^{2} H_{0}^{2}(t)}{2 \omega \zeta^{2} P^{2}(t) k^{\mathbb{P}}}=0 \\
& P_{0}^{\prime}(t)(x+\beta l)+\frac{Q_{0}^{\prime}(t)}{\omega} v+\frac{R_{0}^{\prime}(t)}{\omega}+\left[\left(r-\gamma_{1}-\gamma_{2}+\beta\right)(x+\beta l)+\xi_{1} a_{1}+\xi_{2} a_{2}\right] P_{0}(t) \\
& +\left(\delta^{2} v+a_{1} \eta_{1} n_{1}+a_{2} \eta_{2} n_{2}\right) \frac{H_{0}(t)}{\omega P_{0}(t)}+\kappa(\alpha-v) \frac{Q_{0}(t)}{\omega}-\delta v \sigma \rho \frac{Q_{0}(t)}{\omega} \\
& +\left(\frac{\vartheta P_{0}(t)}{\zeta}-\frac{\vartheta P_{0}^{2}(t)}{P(t) \zeta}\right)(x+\beta l)+\frac{\vartheta P_{0}(t)\left(Q(t)-Q_{0}(t)\right)}{P(t) \zeta \omega} v+\frac{H(t) k^{\mathbb{P}}}{P(t) \omega}-\frac{\vartheta H_{0}(t)}{P(t) \zeta \omega} \\
& -\frac{\vartheta H(t) P_{0}(t)}{P^{2}(t) \zeta \omega}+\frac{\vartheta^{2} H_{0}(t) P_{0}(t)}{P^{2}(t) \zeta^{2} \omega k^{\mathbb{P}}}+\frac{\vartheta P_{0}(t)\left(R(t)-R_{0}(t)\right)}{P(t) \zeta \omega}=0 .
\end{aligned}
$$


By separating variables, we derive the following differential equations:

$$
\begin{aligned}
& H_{0}^{\prime}(t)+\left(r-\gamma_{1}-\gamma_{2}+\beta+\frac{\vartheta}{\zeta}-k^{\mathbb{P}}\right) H_{0}(t)-\left(\frac{\vartheta H_{0}(t)}{P(t) \zeta}-k^{\mathbb{P}}\right) P_{0}(t)=0, \quad H_{0}(T)=1, \\
& P_{0}^{\prime}(t)+\left(r-\gamma_{1}-\gamma_{2}+\beta+\frac{\vartheta}{\zeta}\right) P_{0}(t)-\frac{\vartheta P_{0}^{2}(t)}{P(t) \zeta}=0, \quad P_{0}(T)=1, \\
& Q_{0}^{\prime}(t)-\left(\kappa+\delta \sigma \rho+\frac{\vartheta P_{0}(t)}{P(t) \zeta}\right) Q_{0}(t)+\frac{\vartheta P_{0}(t) Q(t)}{P(t) \zeta}+\delta^{2} \frac{H_{0}(t)}{P_{0}(t)}=0, \quad Q_{0}(T)=0, \\
& F_{0}^{\prime}(t)-\left(\kappa+k^{\mathbb{P}}\right) F_{0}(t)-\frac{\sigma^{2}}{2} Q_{0}^{2}(t)\left(1-\rho^{2}\right)-\frac{\delta \sigma \rho H_{0}(t) Q_{0}(t)}{P_{0}(t)}+\frac{\delta^{2} H_{0}^{2}(t)}{2 P_{0}^{2}(t)}+F(t) k^{\mathbb{P}} \\
& +\left(\frac{\vartheta H_{0}(t)}{P(t) \zeta}-k^{\mathbb{P}}\right)\left(Q(t)-Q_{0}(t)\right)=0, \quad F_{0}(T)=0, \\
& R_{0}^{\prime}(t)+\omega\left(\xi_{1} a_{1}+\xi_{2} a_{2}\right) P_{0}(t)+\kappa \alpha Q_{0}(t)+\left(a_{1} \eta_{1} n_{1}+a_{2} \eta_{2} n_{2}\right) \frac{H_{0}(t)}{P_{0}(t)}-\frac{\vartheta H_{0}(t)}{P(t) \zeta} \\
& -\frac{\vartheta H(t) P_{0}(t)}{P^{2}(t) \zeta}+\frac{\vartheta^{2} H_{0}(t) P_{0}(t)}{P^{2}(t) \zeta^{2} k^{\mathbb{P}}}+\frac{\vartheta P_{0}(t)\left(R(t)-R_{0}(t)\right)}{P(t) \zeta}+\frac{H(t) k^{\mathbb{P}}}{p(t)}=0, \quad R_{0}(T)=0, \\
& G_{0}^{\prime}(t)-k^{\mathbb{P}} G_{0}(t)+k^{\mathbb{P}} G(t)+\omega\left(\xi_{1} a_{1}+\xi_{2} a_{2}\right) H_{0}(t)+\kappa \alpha F_{0}(t)+\left(a_{1} \eta_{1} n_{1}+a_{2} \eta_{2} n_{2}\right. \\
& \left.-\frac{1}{2}\left(b_{1}^{2} n_{1}^{2}+b_{2}^{2} n_{2}^{2}\right)-\lambda \mu_{11} \mu_{21} n_{1} n_{2}\right) \frac{H_{0}^{2}(t)}{P_{0}^{2}(t)}+\left(\frac{\vartheta H_{0}(t)}{P(t) \zeta}-\frac{H(t)}{P(t)} k^{\mathbb{P}}\right)\left(R(t)-R_{0}(t)\right) \\
& +\frac{H^{2}(t) k^{\mathbb{P}}}{2 P^{2}(t)}-\frac{\vartheta H(t) H_{0}(t)}{P^{2}(t) \zeta}+\frac{\vartheta^{2} H_{0}^{2}(t)}{2 \zeta^{2} P^{2}(t) k^{\mathbb{P}}}=0, \quad G_{0}(T)=0 .
\end{aligned}
$$

The solutions to the above equations are as follows:

$$
\begin{aligned}
H_{0}(t)= & P_{0}(t)=e^{\left(r-\gamma_{1}-\gamma_{2}+\beta\right)(T-t)}, \\
Q_{0}(t)= & \left\{\begin{array}{l}
\frac{\delta^{2}}{\kappa+\delta \sigma \rho}\left[1-e^{(t-T)(\kappa+\delta \sigma \rho)}\right], \quad \kappa+\delta \sigma \rho \neq 0, \\
(T-t) \delta^{2}, \quad \kappa+\delta \sigma \rho=0,
\end{array}\right. \\
F_{0}(t)= & e^{\left(\kappa+k^{\mathbb{P}}\right) t} \int_{t}^{T} e^{-\left(\kappa+k^{\mathbb{P}}\right) u\left[-\frac{\sigma^{2}}{2} Q_{0}^{2}(u)\left(1-\rho^{2}\right)-\delta \sigma \rho Q_{0}(u)+\frac{\delta^{2}}{2}+F(u) k^{\mathbb{P}}\right] \mathrm{d} u,} \\
R_{0}(t)= & e^{(\vartheta / \zeta) t} \int_{t}^{T} e^{-(\vartheta / \zeta) u}\left[\frac{\vartheta}{\zeta} R(u)+\omega\left(\xi_{1} a_{1}+\xi_{2} a_{2}\right) P_{0}(u)+\kappa \alpha Q_{0}(u)+a_{1} \eta_{1} n_{1}+a_{2} \eta_{2} n_{2}\right.
\end{aligned}
$$


Noting (B.1), (B.2), (B.13), (B.18)-(B.24), (B.34), and (B.37)-(B.41), the explicit expression of equilibrium strategies and the corresponding equilibrium value function are derived easily. Then, the proof of Theorem 2 is completed.

\section{Data Availability}

The data in this paper can be used publicly.

\section{Conflicts of Interest}

The authors declare that they have no conflicts of interest.

\section{References}

[1] S. Browne, "Optimal investment policies for a firm with a random risk process: exponential utility and minimizing the probability of ruin," Mathematics of Operations Research, vol. 20, no. 4, pp. 937-958, 1995.

[2] H. Yang and L. Zhang, "Optimal investment for insurer with jump-diffusion risk process," Insurance: Mathematics and Economics, vol. 37, no. 3, pp. 615-634, 2005.

[3] L. Bai and H. Zhang, "Dynamic mean-variance problem with constrained risk control for the insurers," Mathematical Methods of Operations Research, vol. 68, no. 1, pp. 181-205, 2008.

[4] C. Hipp and M. Plum, "Optimal investment for insurers," Insurance: Mathematics and Economics, vol. 27, no. 2, pp. 215-228, 2000.

[5] M. Kaluszka, "Optimal reinsurance under mean-variance premium principles," Insurance: Mathematics and Economics, vol. 28, no. 1, pp. 61-67, 2001.

[6] B. Højgaard and M. Taksar, "Optimal proportional reinsurance policies for diffusion models with transaction costs," Insurance: Mathematics and Economics, vol. 22, no. 1, pp. 41-51, 1998.

[7] Y. Cao and N. Wan, "Optimal proportional reinsurance and investment based on Hamilton-Jacobi-bellman equation," Insurance: Mathematics and Economics, vol. 45, no. 2, pp. 157-162, 2009.

[8] X.-L. Zhang, K.-C. Zhang, and X.-J. Yu, "Optimal proportional reinsurance and investment with transaction costs, I: maximizing the terminal wealth, insurance," Mathematics and Economics, vol. 44, no. 3, pp. 473-478, 2009.

[9] M. Gu, Y. Yang, S. Li, and J. Zhang, "Constant elasticity of variance model for proportional reinsurance and investment strategies," Insurance: Mathematics and Economics, vol. 46, no. 3, pp. 580-587, 2010.

[10] H. Zao, X. Rong, and Y. Zhao, "Optimal excess-of-loss reinsurance and investment problem for an insurer with jumpdiffusion risk process under the Heston model," Insurance: Mathematics and Economics, vol. 53, no. 3, pp. 504-514, 2013.

[11] T. Bjork and A. Murgoci, "A general theory of markovian time inconsistent stochastic control problems," Stockholm School of School, Stockholm, Sweden, Working Paper, 2010.

[12] T. Björk, M. Khapko, and A. Murgoci, "On time-inconsistent stochastic control in continuous time," Finance and Stochastics, vol. 21, no. 2, pp. 331-360, 2017.

[13] Y. Zeng and Z. Li, "Optimal time-consistent investment and reinsurance policies for mean-variance insurers," Insurance: Mathematics and Economics, vol. 49, no. 1, pp. 145-154, 2011.
[14] Z. Li, Z. Yan, and Y. Lai, "Optimal time-consistent investment and reinsurance strategies for insurers under Heston's SV model," Insurance: Mathematics and Economics, vol. 51, no. 1, pp. 191-203, 2012.

[15] Z. Liang and M. Song, "Time-consistent reinsurance and investment strategies for mean-variance insurer under partial information," Insurance: Mathematics and Economics, vol. 65, pp. 66-76, 2015.

[16] L. Bai, J. Cai, and M. Zhou, "Optimal reinsurance policies for an insurer with a bivariate reserve risk process in a dynamic setting," Insurance: Mathematics and Economics, vol. 53, no. 3 , pp. 664-670, 2013.

[17] Z. Liang and K. C. Yuen, "Optimal dynamic reinsurance with dependent risks: variance premium principle," Scandinavian Actuarial Journal, vol. 2016, no. 1, pp. 1-19, 2014.

[18] K. C. Yuen, Z. Liang, and M. Zhou, "Optimal proportional reinsurance with common shock dependence," Insurance: Mathematics and Economics, vol. 64, pp. 1-13, 2015.

[19] Z. Ming, Z. Liang, and C. Zhang, "Optimal mean-variance reinsurance with common shock dependence," The ANZIAM Journal, vol. 58, no. 2, pp. 162-181, 2016.

[20] J. Bi, Z. Liang, and F. Xu, "Optimal mean-variance investment and reinsurance problems for the risk model with common shock dependence," Insurance: Mathematics and Economics, vol. 70, pp. 245-258, 2016.

[21] J. Bi and K. Chen, "Optimal investment-reinsurance problems with common shock dependent risks under two kinds of premium principles," RAIRO-Operations Research, vol. 53, no. 1, pp. 179-206, 2019.

[22] I. Elsanosi, B. Øksendal, and A. Sulem, "Some solvable stochastic control problems with delay," Stochastics and Stochastic Reports, vol. 71, no. 1-2, pp. 69-89, 2000.

[23] B. Øksendal and A. Sulem, A Maximum Principle for Optimal Control of Stochastic Systems with Delay, with Applications to Finance, pp. 64-79, IOS Press, Amsterdam, Netherlands, 2001.

[24] D. David, "Optimal control of stochastic delayed systems with jumps," working paper or preprint, 2008.

[25] S. Federico, B. Goldys, and F. Gozzi, "HJB equations for the optimal control of differential equations with delays and state constraints, I: regularity of viscosity solutions," SIAM Journal on Control and Optimization, vol. 48, no. 8, pp. 4910-4937, 2010.

[26] M. H. Chang, T. Pang, and Y. Yang, "A stochastic portfolio optimization model with bounded memory," Mathematics of Operations Research, vol. 36, no. 4, pp. 604-619, 2011.

[27] S. Federico, "A stochastic control problem with delay arising in a pension fund model," Finance and Stochastics, vol. 15, no. 3, pp. 421-459, 2011.

[28] Y. Shen and Y. Zeng, "Optimal investment-reinsurance with delay for mean-variance insurers: a maximum principle approach," Insurance: Mathematics and Economics, vol. 57, pp. 1-12, 2014.

[29] T. R. Bielecki and I. Jang, "Portfolio optimization with a defaultable security," Asia-Pacific Financial Markets, vol. 13, no. 2, pp. 113-127, 2006.

[30] J. Driessen, "Is default event risk priced in corporate bonds?" The Review of Financial Studies, vol. 18, no. 1, pp. 165-195, 2004.

[31] D. Duffie and K. J. Singleton, Credit Risk. Pricing, Measurement, and Management, Princeton University Press, Princeton, NJ, USA, 2003.

[32] S. Kusuoka, "A remark on default risk models," Advances in Mathematical Economics, vol. 1, pp. 69-82, 1999. 
[33] F. Yu, "Modeling expected return on defaultable bonds," The Journal of Fixed Income, vol. 12, no. 2, pp. 69-81, 2002.

[34] H. Zhu, C. Deng, S. Yue, and Y. Deng, "Optimal reinsurance and investment problem for an insurer with counterparty risk," Insurance: Mathematics and Economics, vol. 61, pp. 242-254, 2015. 